\title{
Longer-queue-serve-first System
}

\author{
by \\ Wenzhe Ye
}

A thesis submitted to the Faculty of Graduate and Postdoctoral Affairs

in partial fulfillment of the requirements for the degree of

Master of Science

in

Probability and Statistics

\author{
Carleton University \\ Ottawa, Ontario
}

@ 2012

Wenzhe Ye 
Library and Archives

Canada

Published Heritage

Branch

395 Wellington Street

Ottawa ON K1A ON4

Canada
Bibliothèque et

Archives Canada

Direction du

Patrimoine de l'édition

395 , rue Wellington

Ottawa ON K1A ON4

Canada
Your file Votre référence

ISBN: 978-0-494-91510-3

Our file Notre référence

ISBN: 978-0-494-91510-3
NOTICE:

The author has granted a nonexclusive license allowing Library and Archives Canada to reproduce, publish, archive, preserve, conserve, communicate to the public by telecommunication or on the Internet, loan, distrbute and sell theses worldwide, for commercial or noncommercial purposes, in microform, paper, electronic and/or any other formats.

The author retains copyright ownership and moral rights in this thesis. Neither the thesis nor substantial extracts from it may be printed or otherwise reproduced without the author's permission.
AVIS:

L'auteur a accordé une licence non exclusive permettant à la Bibliothèque et Archives Canada de reproduire, publier, archiver, sauvegarder, conserver, transmettre au public par télécommunication ou par l'Internet, prêter, distribuer et vendre des thèses partout dans le monde, à des fins commerciales ou autres, sur support microforme, papier, électronique et/ou autres formats.

L'auteur conserve la propriété du droit d'auteur et des droits moraux qui protege cette thèse. $\mathrm{Ni}$ la thèse ni des extraits substantiels de celle-ci ne doivent être imprimés ou autrement reproduits sans son autorisation.
In compliance with the Canadian Privacy Act some supporting forms may have been removed from this thesis.

While these forms may be included in the document page count, their removal does not represent any loss of content from the thesis.
Conformément à la loi canadienne sur la protection de la vie privée, quelques formulaires secondaires ont été enlevés de cette thèse.

Bien que ces formulaires aient inclus dans la pagination, il n'y aura aucun contenu manquant. 


\begin{abstract}
This thesis focuses on a model which has great potential for application, namely the longer-queue-serve-first queueing model with unequal arrival rates. Our goal is to obtain the tail asymptotic behavior for the stationary distribution, which is useful in computing performance measures observed over a long period of time. We demonstrate in detail how to apply the kernel method with multiple kernel equations to locate dominant singularities, and to determine the detailed behaviors of unknown generating functions at their dominant singularities according to analytic characteristics. The main contributions made in this thesis are: (1) characterization of exact tail asymptotic behavior of this model, which has not be given in the literature; (2) application of the kernel method to multiple kernel equations, which extends the possibility of solving random walks in the quarter plane into that in the half plane.
\end{abstract}




\section{Acknowledgements}

First and foremost, I would like to thank my supervisors Dr. Yiqiang Zhao from Carleton University and Dr. Hui Li from Mount Saint Vincent University for their patient guidance. I appreciate Professor Zhao's helpful guidance and stimulating encouragement through out the research work and thank him for introducing me to the world of exact tail asymptotic analysis and queueing theory. I would also like to express my gratitude to Professor Li for her grateful professional help on my research and for her valuable comments, suggestions and support. Without their wisdom and expertise, this thesis would not have been completed.

Secondly, I would like to thank Dr. Evangelos Kranakis, Dr. Gennady Shaikhet and Dr. Rafal Kulik for serving on my defense committee and for taking time to read my thesis. Thanks are also due to all the brilliant faculty members of the School of Mathematics and Statistics, and to Carleton University, for offering such a wonderful program. Finally, I would like to give my special thanks to my family for supporting me, encouraging me, inspiring me. 


\section{Contents}

Abstract $\quad$ ii

Acknowledgements

1 Introduction 1

1.1 Motivations ......................... 1

1.2 Background ........................... 3

1.2.1 Tail asymptotics of probabilities . . . . . . . . . . . 3

1.2.2 Taubarian-like theorems ............... 4

1.3 Contributions ....................... 6

1.4 Organization ....................... 7

2 Longer-queue-serve-first Model and Generating Functions 9

2.1 Preemptive longer-queue-serve-first model f . . . . . . . . . . . 9

2.2 Generating functions . . . . . . . . . . . . . . 13

3 Tail Asymptotic Analysis along the $y$-Direction 20

4 Dominant Singularity Analysis of $\varphi_{j}(x)$

4.1 Kernel method . . . . . . . . . . . . . . . . . . 24

4.2 Dominant singularity analysis of $\varphi_{j}(x) \ldots \ldots 26$ 
5 Tail Asymptotic Analysis along the $x$-Direction 36

5.1 Asymptotic analysis of $\varphi_{0}(x) \ldots \ldots \ldots \ldots \ldots \ldots$

5.2 Asymptotic analysis of $\varphi_{j}(x), j \neq 0 \ldots \ldots \ldots \ldots \ldots \ldots$

5.3 Tail asymptotic results $\ldots \ldots \ldots \ldots \ldots \ldots$

$\begin{array}{lll}6 & \text { Conclusion } & 48\end{array}$

$\begin{array}{ll}\text { Bibliography } & 49\end{array}$ 


\section{Chapter 1}

\section{Introduction}

\subsection{Motivations}

Multidimensional queueing systems related to networks have been introduced and widely studied. Performance measures over a long period of time aid practitioners in designing systems and evaluating their performance. Usually, our primary interest is the stationary distribution, from which performance measures over a long period of time can be computed.

However, except for special cases, obtaining a simple analytic or closed form solution of the stationary distribution of even a simple stochastic network is not feasible. Thus, we may turn our focus to some characteristics which are still useful in assessing performance, which include tail asymptotic behavior of the stationary distribution. Though the analysis of tail asymptotics is still a hard problem, it is greatly simpler than working with the stationary distribution itself. This motivates the study of exact tail asymptotic behavior of the stationary distribution.

In this thesis, we consider exact tail asymptotic properties of a longer-queue-serve-first preemptive model with unequal arrival rates (see Chapter 2 for the detailed model description). Longer-queue-serve-first (or longest-queue-serve-first) models vary in 


\begin{tabular}{|c|c|c|c|c|}
\hline model & $\begin{array}{c}\text { preemption/ } \\
\text { non-preemption }\end{array}$ & dimensions & $\begin{array}{c}\text { arrival } \\
\text { rates }\end{array}$ & $\begin{array}{c}\text { service time } \\
\text { distribution }\end{array}$ \\
\hline Cohen [16] & non-preemption & 2 & unequal & general \\
Zheng and Zipkin [18] & preemption & 2 & equal & exponential \\
Flatto [15] & preemption & 2 & equal & exponential \\
Zipkin [19] & preemption & $\geq 2$ & equal & exponential \\
Adan [17] & preemption & $\geq 2$ & equal & exponential \\
\hline
\end{tabular}

Table 1.1: Models in the literature

the literature, with variation on preemption/non-preemption description, queueing dimensions, unequal/equal Poisson arrival rates, exponential/general service time distributions; see Table 1.1 for a summary.

In the literature about longer-queue-serve-first (or longest-queue-serve-first) models, authors have used complex analysis to determine the generating function, the stationary distribution, or tail asymptotic properties, such as Flatto [15] and Cohen [16]; and have made queueing comparisons to obtain performance bounds, such as Adan [17]. The model with equal Poisson arrival rates and exponential service time has been studied in Zheng and Zipkin [18], where an explicit solution of the distribution of the difference between queue lengths is obtained directly from the balance equations. In Flatto [15], the same model has been analyzed. The approach via generating functions is used to derive exact tail asymptotic formulas and to study the interdependence of the two queue lengths. For the model with more than two queues, Zipkin [19] proposed an approximation method. The non-preemptive model with unequal Poisson arrival rates and general service time distribution has been studied in Cohen [16], via the use of a translation into a Riemann boundary value problem.

For the tail asymptotic analysis, four approaches are normally used in the literature, namely brute force approach, large deviations approach, Markov additive approach and analytic function approach. A detailed literature review of these four approaches can be found in Miyazawa [9]. 


\subsection{Background}

Tail asymptotics along coordinate directions and Taubarian-like theorems are introduced here. Analytic continuation, singularities, branch points, etc, are standard concepts in analysis. For more details, readers may refer to [8] and [2].

\subsubsection{Tail asymptotics of probabilities}

In this thesis, we consider the exact tail asymptotics behavior of a 2-dimensional model (with state space $S=\mathbb{Z}_{+} \times \mathbb{Z}=\{(i, j) ; i=0,1,2, \ldots, j=0, \pm 1, \pm 2, \ldots\}$ ) along coordinate directions.

Definition 1.1 Let $(X, Y)$ be a 2-dimensional random vector with $X=0,1,2, \ldots$ and $Y=0, \pm 1, \pm 2, \ldots$. The exact tail asymptotic functions of the tail probabilities along three directions: along the $x$ direction and along the $y$ direction (either the $y$ positive direction or the $y$ negative direction), can be defined as follows:

(1) The tail probability along the $x$ direction with the other dimension fixed, is said to have exact tail asymptotic function if it can be written as:

$$
\lim _{x \rightarrow \infty} \frac{P(X=x, Y=y)}{h(x)}=b(y), \text { for a given } y, y=0, \pm 1, \pm 2, \ldots
$$

where $b$ is a positive constant and $h$ is a positive-valued function.

(2) The tail probability along the $y$ positive direction with the other dimension fixed, is said to have exact tail asymptotic function if it can be written as:

$$
\lim _{y \rightarrow \infty} \frac{P(X=x, Y=y)}{h(y)}=b(x), \text { for a given } x, x=0,1,2, \ldots
$$

where $b$ is a positive constant and $h$ is a positive-valued function.

The tail probability along the $y$ negative direction with the other dimension fixed, is 
said to have exact tail asymptotic function if it can be written as:

$$
\lim _{y \rightarrow-\infty} \frac{P(X=x, Y=y)}{h(y)}=b(x), \text { for a given } x, x=0,1,2, \ldots
$$

where $b$ is a positive constant and $h$ is a positive-valued function.

Remark 1.1 A generalized version of the definitions of exact tail asymptotic functions along any direction for a p-dimension model is provided in Miyazawa [9].

\subsubsection{Taubarian-like theorems}

The following theorem is from Bender [10], which can also be found in Flajolet and Sedgewick [11].

Theorem 1.1 (With multiple dominant singularities) Suppose $A(z)=$ $\sum_{n \geq 0} a_{n} z^{n}$ is analytic near zero. Let $\alpha_{k}, k=1, \ldots, m$, be all singular points on its circle $|z|=c_{r}>0$ of convergence, and for each $k$, there exists $\omega_{k}$ and $g_{k}$, such that

$$
\lim _{z \rightarrow \alpha_{k}}\left(1-z / \alpha_{k}\right)^{\omega_{k}} A(z)=g_{k}
$$

where $\omega_{k}$ is a complex number not equal to $0,1,2,3, \ldots$, and $g_{k}$ is a non-zero constant. Then,

$$
a_{n} \sim \frac{1}{n} \sum_{k} \frac{g_{k} n^{\Re\left(\omega_{k}\right)}}{\Gamma\left(\Re\left(\omega_{k}\right)\right)\left[\Re\left(\alpha_{k}\right)\right]^{n}}
$$

where $\Re(z)$ is the real part of $z$.

Theorem 1.2 (With a single dominant singularity) Suppose $A(z)=\sum_{n \geq 0} a_{n} z^{n}$ is analytic in $\triangle(\phi, \epsilon) \backslash\{1\}$, where $\triangle(\phi, \epsilon)=\{z:|z| \leq 1+\epsilon,|\operatorname{Arg}(z-1)| \geq \phi\}$ with 
$\epsilon>0,0<\phi<\pi / 2$, and

$$
A(z) \sim g(1-z)^{\omega} \quad \text { as } z \rightarrow 1 \text { in } \triangle(\phi, \epsilon)
$$

for some complex number $\omega$, as $n \rightarrow \infty$, (i) if $\omega \notin\{0,1,2, \ldots\}$, then

$$
a_{n} \sim \frac{g}{\Gamma(-w)} n^{-\omega-1}
$$

(ii) if $\omega$ is a nonnegative integer, then

$$
a_{n}=o\left(n^{-\omega-1}\right)
$$

Tauberian-like theorems offer a possible method to obtain exact tail asymptotics with the following procedure: firstly, locate the dominant singularities of an unknown generating function, namely $\alpha_{k}, k=1, \ldots, m$; secondly, find $\omega_{k}$ such that for each $\alpha_{k}$, $k=1, \ldots, m, \lim _{z \rightarrow \alpha_{k}}\left(1-z / \alpha_{k}\right)^{\omega_{k}} A(z)$ exists. In this thesis, there is only one dominant singularity and the second version of the Tauberian-like theorem is applied.

Theorem 1.1 and Theorem 1.2 are referred to as Tauberian-like theorems instead of Tauberian theorems in the thesis. This is because one of the conditions in classical Tauberian theorems is monotonicity of the coefficients. In our case, there are an unknown sequence of probabilities on which we do not have monotonicity information. In the Tauberian-like theorems, this condition is replaced by some extra requirements on the analyticity of the unknown function which can often be verified. In Theorem 1.2, the function is required to be analytic on the region $\triangle(\phi, \epsilon) \backslash\{1\}$, which is an indented disk shown in Fig 1.1.

Remark 1.2 If $A(z)=\sum_{n \geq 0} a_{n} z^{n}$ (or $\left.A(z)=\sum_{n \geq 1} a_{n} z^{n}\right), k=1, \alpha_{1} \in \mathbb{R}$ and 


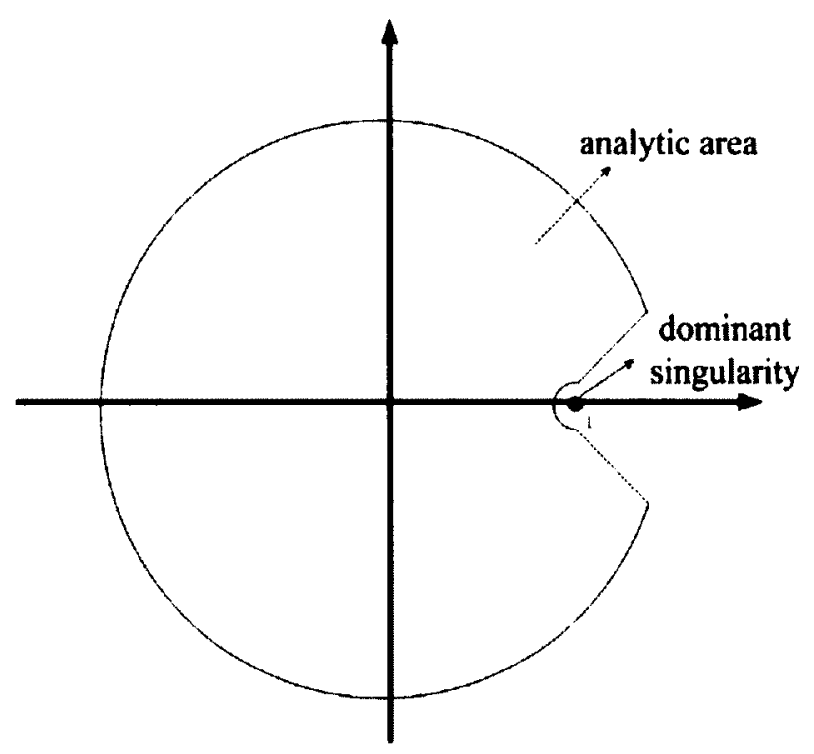

Figure 1.1: Indented analytic area

$\omega_{1} \in \mathbb{R}$, then

$$
a_{n} \sim \frac{g_{1} n^{\omega_{1}-1}}{\Gamma\left(\omega_{1}\right)\left(\alpha_{1}\right)^{n}}
$$

Remark 1.3 If $A(z)=\sum_{n \geq 1} a_{n} z^{n}, k=1, \alpha_{1} \in \mathbb{R}, \omega_{1} \in \mathbb{R}$ and $\lim _{z \rightarrow \alpha_{1}}\left(1-z / \alpha_{1}\right)^{\omega_{1}} A(z)^{\prime}=g_{k}$, then

$$
a_{n} \sim \frac{g_{1} n^{\omega_{1}-2}}{\Gamma\left(\omega_{1}\right)\left(\alpha_{1}\right)^{n-1}}
$$

Proof. Since $A(z)^{\prime}=\sum_{n \geq 1} n a_{n} z^{n-1}=\sum_{n \geq 0}\left((n+1) a_{n+1}\right) z^{n}$, we have $(n+1) a_{n+1} \sim$ $\frac{g_{1} n^{\omega_{1}-1}}{\Gamma\left(\omega_{1}\right)\left(\alpha_{1}\right)^{n}}$. Thus $a_{n} \sim \frac{g_{1}(n-1)^{\omega_{1}-1}}{n \Gamma\left(\omega_{1}\right)\left(\alpha_{1}\right)^{n-1}} \sim \frac{g_{1} n^{\omega_{1}-2}}{\Gamma\left(\omega_{1}\right)\left(\alpha_{1}\right)^{n-1}}$.

\subsection{Contributions}

This thesis focuses on a longer-queue-serve-first queue model with unequal arrival rates, which has great potential for application. Our goal is to obtain asymptotic behavior for the stationary distribution, which is helpful for computing the performance 
measures observed over a long period of time.

The main contributions made in this thesis are: (1) characterization of exact tail asymptotic behavior of this model; and (2) application of the kernel method to multiple kernel equations, which extends the possibility of solving random walks in the quarter plane into that in the half plane. The tail asymptotic behavior of the model with equal arrival rates has been studied in Flatto [15], with a method using the concepts of Riemann surfaces and uniformization theory, while tail asymptotic behavior of the model with unequal arrival rates has not been given in the literature; the latter case, for which we use the kernel method, is the focus of this thesis. The kernel method with one kernel equation can be used to obtain tail asymptotic properties of random walks in the quarter plane, for example, see $\mathrm{Li}$ and Zhao $[3,4,5,6,7]$, while work using multiple kernel equations is very limited and has not been reported in the literature.

\subsection{Organization}

This thesis is organized as follows: Chapters 1 and 2 form the first part of the thesis. Chapter 1 provides a brief introduction to the problem, presenting some background to the subsequent chapters. In Chapter 2, a longer-queue-serve-first model is formally introduced and presented as a random walk in the half plane. Then generating functions are defined, and two series of important relationships among these generating functions are obtained. One consists of recursive equations and the other provides fundamental forms.

With all the material available, the remaining part focuses on how to apply the kernel method and the Tauberian-like theorems to obtain exact tail asymptotic properties. Chapter 3 concentrates on the exact tail asymptotic analysis along the $y$-direction, while Chapter 4 and Chapter 5 are combined to complete the exact tail asymptotic 
analysis along the $x$-direction.

In Chapter 3, according to the recursive relationship derived in Chapter 2, we can find explicit expressions for the generating function $\psi_{i}^{+}(y), \psi_{i}^{-}(y), i=0,1,2, \ldots$ (see (2.12) and (2.13) for the definition), from which dominant singularities are located and behavior of the unknown generating function at the dominant singularity is studied. The object of Chapter 4 is to demonstrate how to apply the kernel method to locate the dominant singularity of $\varphi_{j}(x), j=0, \pm 1, \pm 2, \ldots$, (see (2.11) for the definition). In Chapter 5 , the behavior of $\varphi_{j}(x), j=0, \pm 1, \pm 2, \ldots$, at the dominant singularity is detailed and tail asymptotic results along the $x$-direction are given. 


\section{Chapter 2}

\section{Longer-queue-serve-first Model}

\section{and Generating Functions}

A continuous-time longer-queue-serve-first model described in this chapter is the focus of this thesis. This queueing system consists of two queues and one server, following longer-queue-serve-first policy with preemption. The original model $\left\{\left(\widetilde{L}_{1}(t), \widetilde{L}_{2}(t)\right)\right\}$ and a modified model $\left\{\left(\min \left\{\widetilde{L}_{1}(t), \widetilde{L}_{2}(t)\right\}, \widetilde{L}_{2}(t)-\widetilde{L}_{1}(t)\right)\right\}$ are introduced, where $\widetilde{L}_{1}(t)$ and $\widetilde{L}_{2}(t)$ are the numbers of customers in the two queues including the one in service at time $t$. After the model description, generating functions are defined. According to balance equations, two kinds of important relationships among these generating functions are obtained: one is a set of linear homogeneous second order recursive equations, and the other is a set of fundamental forms.

\subsection{Preemptive longer-queue-serve-first model}

The continuous-time longer-queue-serve-first model described in this chapter consists of two queues and one server, following longer-queue-serve-first policy with preemption. Customers arrive to these two queues according to two independent Poisson processes with rates $\lambda_{1}$ and $\lambda_{2}$ respectively, and the service time is exponen- 


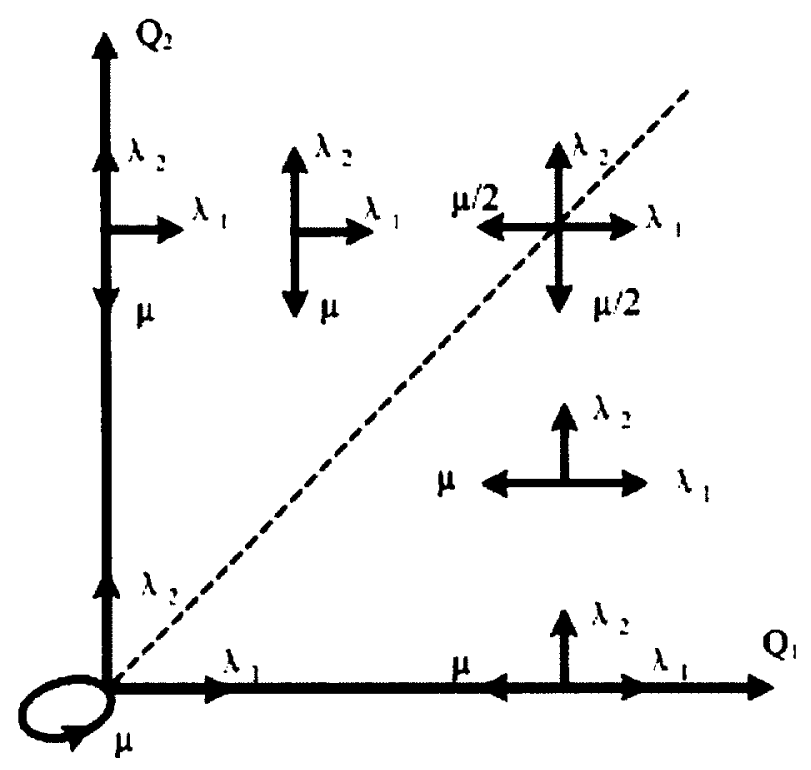

Figure 2.1: Transitions of $\widetilde{\mathbf{P}}$

tially distributed with the mean service time $\frac{1}{\mu}$. Without loss of generality, assume $\lambda_{1}+\lambda_{2}+\mu=1$.

According to the longer-queue-serve-first policy, the server works on the longer queue when queue lengths are not equal and chooses either one to serve with probability $\frac{1}{2}$ when they are of equal length. The service policy is preemptive, which means whenever the queue not being served becomes the longer one, service will be interrupted and switches to the new longer queue.

Let $\widetilde{L}_{1}(t)$ and $\widetilde{L}_{2}(t)$ be the numbers of customers in the two queues including the one in service at time $t .\left\{\left(\widetilde{L}_{1}(t), \widetilde{L}_{2}(t)\right)\right\}$ is a continuous-time Markov chain whose state space is $\widetilde{S}=\mathbb{Z} \times \mathbb{Z}=\{(i, j) ; i, j=0,1,2, \ldots\}$, which is stable if and only if $\mu>\lambda_{1}+\lambda_{2}$. Let $\widetilde{\mathbf{Q}}$ be the infinitesimal generator of the continuous-time Markov chain $\left\{\left(\widetilde{L}_{1}(t), \widetilde{L}_{2}(t)\right)\right\}$. Then, $\widetilde{\mathbf{P}}=I+\widetilde{\mathbf{Q}}$ is the transition probability matrix for the uniformized discrete-time Markov chain and the transition diagram of $\widetilde{\mathbf{P}}$ is shown in the Fig 2.1.

We notice that $\left\{\left(\widetilde{L}_{1}(t), \widetilde{L}_{2}(t)\right)\right\}$ is not a random walk in the quarter plane in the usual sense, since instead of one it has two regions on which transitions are homoge- 
neous. Motivated from this, we define a different Markov chain $\left\{\left(L_{1}(t), L_{2}(t)\right)\right\}$, where $L_{1}(t)=\min \left\{\widetilde{L}_{1}(t), \widetilde{L}_{2}(t)\right\}$ and $L_{2}(t)=\widetilde{L}_{2}(t)-\widetilde{L}_{1}(t) .\left\{\left(L_{1}(t), L_{2}(t)\right)\right\}$ is a continuoustime Markov chain with state space $S=\mathbb{Z}_{+} \times \mathbb{Z}=\{(i, j) ; i=0,1,2, \ldots, j=$ $0, \pm 1, \pm 2, \ldots\}$, which is a random walk in the half plane. $\widetilde{L}_{2}(t)-\widetilde{L}_{1}(t)$ is used instead of $\left|\widetilde{L}_{2}(t)-\widetilde{L}_{1}(t)\right|$ here, because the process $\left\{\left(\min \left\{\widetilde{L}_{1}(t), \widetilde{L}_{2}(t)\right\},\left|\widetilde{L}_{2}(t)-\widetilde{L}_{1}(t)\right|\right)\right\}$ will no longer be a Markov chain. $\left(\widetilde{L}_{1}(t), \widetilde{L}_{2}(t)\right)$ is equivalent to $\left(L_{1}(t), L_{2}(t)\right)$ in the sense that $\left(\widetilde{L}_{1}(t), \widetilde{L}_{2}(t)\right)$ and $\left(L_{1}(t), L_{2}(t)\right)$ are uniquely determined by each other.

Let the system be in state $\left(L_{1}(t)=m, L_{2}(t)=n\right)$. For $m>0$ and $n>0$, suppose the next transition is due to an arrival, then an arrival on the shorter queue leads to $\left(L_{1}(t+1)=m+1, L_{2}(t+1)=n-1\right)$, and one on the longer queue leads to $\left(L_{1}(t+1)=m, L_{2}(t+1)=n+1\right)$. Also, suppose the next transition is due to a departure, then the departure from the longer queue leads to $\left(L_{1}(t)=m, L_{2}(t)=n-1\right)$. There is no departure from the shorter queue according to the longer-queue-servefirst policy. For other values of $m$ and $n$, the transition situations can be similarly analyzed. Let $\mathbf{Q}$ be the infinitesimal generator of the continuous-time Markov chain $\left\{\left(L_{1}(t), L_{2}(t)\right)\right\}$. Then, $\mathbf{P}=I+\mathbf{Q}$ is the transition probability matrix for the uniformized discrete-time Markov chain; the transition diagram of $\mathbf{P}$ is shown in the Fig 2.2 .

We denote by $\widetilde{\pi}_{i, j}$ the limiting probabilities for the process $\left\{\left(\widetilde{L}_{1}(t), \widetilde{L}_{2}(t)\right)\right\}$, and by $\pi_{i, j}$ the limiting probabilities for the process $\left\{\left(L_{1}(t), L_{2}(t)\right)\right\}$. The stability condition of $\left\{\left(L_{1}(t), L_{2}(t)\right)\right\}$ is the same as that of $\left\{\left(\widetilde{L}_{1}(t), \widetilde{L}_{2}(t)\right)\right\}$, namely, $\mu>\lambda_{1}+\lambda_{2}$. These two Markov chains have the same stability condition. To see this, assume $\left\{\left(\widetilde{L}_{1}(t), \widetilde{L}_{2}(t)\right)\right\}$ is stable (ergodic), which is equivalent to the existence of the unique probability solution to its stationary equations $\tilde{\pi}=\tilde{\pi} \widetilde{\mathbf{P}}$. These same equations can be also viewed as the stationary equations of $\left\{\left(L_{1}(t), L_{2}(t)\right)\right\}$ since there is a one-to-one correspondence between variables $\left(\widetilde{L}_{1}(t), \widetilde{L}_{2}(t)\right)$ and variables $\left(L_{1}(t), L_{2}(t)\right)$.

For convenience, $S$ is partitioned as $S=S_{.,+} \cup S_{.,-} \cup S_{+, 0} \cup S_{0,0}$, or $S=S_{+,+} \cup S_{+, 0} \cup$ 


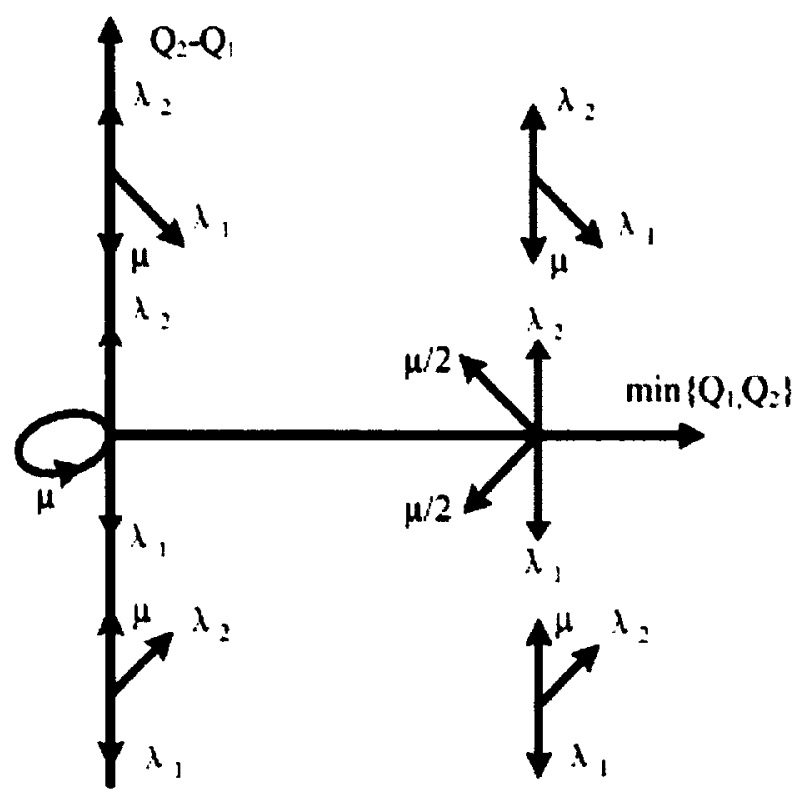

Figure 2.2: Transitions of $\mathbf{P}$

$S_{+,-} \cup S_{0,+} \cup S_{0,0} \cup S_{0,-}$ where

$$
\begin{aligned}
& S_{+,+}=\{(m, n) ; m=1,2, \ldots n=1,2, \ldots\}, \\
& S_{+, 0}=\{(m, 0) ; m=1,2, \ldots\}, \\
& S_{+,-}=\{(m, n) ; m=1,2, \ldots n=-1,-2, \ldots\}, \\
& S_{0,+}=\{(0, n) ; n=1,2, \ldots\}, \quad S_{0,0}=\left\{(0,0\}, \quad S_{0,-}=\{(0, n) ; n=-1,-2, \ldots\},\right.
\end{aligned}
$$

and

$$
S_{.,+}=S_{0,+} \cup S_{+,+}, \quad S_{.,-}=S_{0,-} \cup S_{+,-} .
$$


The balance equations based on the transition diagram in Fig 2.2 are the following.

$$
\begin{aligned}
\pi_{i, j} & =\lambda_{1} \pi_{i-1, j+1}+\lambda_{2} \pi_{i, j-1}+\mu \pi_{i, j+1}, \quad i \geq 1, \quad j \geq 2, \\
\pi_{i, 1} & =\lambda_{1} \pi_{i-1,2}+\lambda_{2} \pi_{i, 0}+\mu \pi_{i, 2}+\frac{\mu}{2} \pi_{i+1,0}, \quad i \geq 1, \\
\pi_{i, 0} & =\lambda_{1} \pi_{i-1,1}+\lambda_{2} \pi_{i-1,-1}+\mu \pi_{i, 1}+\mu \pi_{i,-1}, \quad i \geq 1, \\
(1-\mu) \pi_{0,0} & =\mu \pi_{0,1}+\mu \pi_{0,-1}, \\
\pi_{0, j} & =\lambda_{2} \pi_{0, j-1}+\mu \pi_{0, j+1}, \quad j \geq 2, \\
\pi_{0,1} & =\lambda_{2} \pi_{0,0}+\mu \pi_{0,2}+\frac{\mu}{2} \pi_{1,0}, \\
\pi_{0,-1} & =\lambda_{1} \pi_{0,0}+\mu \pi_{0,-2}+\frac{\mu}{2} \pi_{1,0}, \\
\pi_{0, j} & =\lambda_{1} \pi_{0, j+1}+\mu \pi_{0, j-1}, \quad j \leq-2, \\
\pi_{i,-1} & =\lambda_{2} \pi_{i-1,-2}+\lambda_{1} \pi_{i, 0}+\mu \pi_{i,-2}+\frac{\mu}{2} \pi_{i+1,0}, \quad i \geq 1, \\
\pi_{i, j} & =\lambda_{2} \pi_{i-1, j-1}+\lambda_{1} \pi_{i, j+1}+\mu \pi_{i, j-1}, \quad i \geq 1, \quad j \leq-2 .
\end{aligned}
$$

\subsection{Generating functions}

Analytic function approach is one of the possible approaches to derive the multidimensional tail asymptotics. This approach is to apply the theory of analytic functions to fundamental forms. Before we give fundamental forms, generating functions need to be defined.

Define generating functions as follows:

$$
\begin{aligned}
\varphi_{j}(x) & =\sum_{i=1}^{+\infty} \pi_{i, j} x^{i}, \quad|x|<1, \quad j=0, \pm 1, \pm 2, \ldots \\
\psi_{i}^{+}(y) & =\sum_{j=1}^{+\infty} \pi_{i, j} y^{j}, \quad|y|<1, \quad i=0,1,2, \ldots ; \\
\psi_{i}^{-}(y) & =\sum_{j=-1}^{-\infty} \pi_{i, j} y^{-j}, \quad|y|<1, \quad i=0,1,2, \ldots
\end{aligned}
$$




$$
\pi_{p, q}(x, y)= \begin{cases}\sum_{(i, j) \in S_{p, q}} \pi_{i, j} x^{i} y^{j}, \quad|x|<1,|y|<1, \quad \text { if } p \in\{.,+, 0\}, & q \in\{+, 0\}, \\ \sum_{(i, j) \in S_{p, q}} \pi_{i, j} x^{i} y^{-j}, \quad|x|<1,|y|<1, & \text { if } p \in\{.,+, 0\}, \quad q=-.\end{cases}
$$

Remark $2.1 \psi_{i}^{-}(y), i=0,1,2, \ldots$, is defined as $\sum_{j=-1}^{-\infty} \pi_{i, j} y^{-j}$, instead of $\sum_{j=-1}^{-\infty} \pi_{i, j} y^{j}$, and $\pi_{p, q}(x, y), p \in\{.,+, 0\}, q=-$, is defined as $\sum_{(i, j) \in S_{p, q}} \pi_{i, j} x^{i} y^{-j}$, instead of $\sum_{(i, j) \in S_{p, q}} \pi_{i, j} x^{i} y^{j}$ in this thesis. In this way, when we add two kernel equations (see (2.23) and (2.24) for the detail), some terms will be cancelled out, which makes our tail asymptotic analysis along the $x$-direction easier. Also, we will see that we do not need to infer $\pi_{i, j}$ directly from $\pi_{p, q}(x, y)$, not to use $\psi_{i}^{+}(y)$ and $\psi_{i}^{-}(y)$ together to obtain tail asymptotics either. Therefore, we can still separate $\pi_{i, j}$ and $\pi_{i,-j}$ apart.

Remark 2.2 We also use $\varphi_{j}(x), j=0, \pm 1, \pm 2, \ldots$, for the analytic continued function of $\sum_{i=1}^{+\infty} \pi_{i, j} x^{i}$ on $\mathbb{C}_{x}$, where $\mathbb{C}_{x}$ is the complex plane for $x . \psi_{i}^{+}(y)$ (or $\psi_{i}^{-}(y)$ ) is also for the analytic continued function of $\sum_{j=1}^{+\infty} \pi_{i, j} y^{j}$ (or $\sum_{j=-1}^{-\infty} \pi_{i, j} y^{-j}$ ) on $\mathbb{C}_{y}$, where $\mathbb{C}_{y}$ is the complex plane for $y$.

Using balance equations of the random walk, we can obtain recursive relationships for $\psi_{i}^{+}(y), \psi_{i}^{-}(y), i=0,1,2, \ldots$ and $\varphi_{j}(y), j=0, \pm 1, \pm 2, \ldots$ These recursive relationships enable us to work out expressions of $\psi_{i}^{+}(y)$ and $\psi_{i}^{-}(y)$, and derive tail asymptotic properties along the $x$-direction after we obtain the tail asymptotics for $\pi_{n, 0}$, with large $n$. 
Lemma 2.1 1. The recursive relationship for $\psi_{i}^{+}(y), i=0,1,2, \ldots$, can be listed as:

$$
\begin{gathered}
\left(\mu y^{-1}-1+\lambda_{2} y\right) \psi_{0}^{+}(y)=-\left(\frac{\mu}{2} \pi_{1,0}+\lambda_{2} \pi_{0,0}\right) y+\mu \pi_{0,1}, \\
\left(\mu y^{-1}-1+\lambda_{2} y\right) \psi_{i}^{+}(y)+\lambda_{1} y^{-1} \psi_{i-1}^{+}(y)=-\left(\frac{\mu}{2} \pi_{i+1,0}+\lambda_{2} \pi_{i, 0}\right) y+\mu \pi_{i, 1}, \\
i \geq 1
\end{gathered}
$$

2. The recursive relationship for $\psi_{i}^{-}(y), i=0,1,2, \ldots$, can be listed as:

$$
\begin{gathered}
\left(\mu y^{-1}-1+\lambda_{1} y\right) \psi_{0}^{-}(y)=-\left(\frac{\mu}{2} \pi_{1,0}+\lambda_{1} \pi_{0,0}\right) y+\mu \pi_{0,-1}, \\
\left(\mu y^{-1}-1+\lambda_{1} y\right) \psi_{i}^{-}(y)+\lambda_{2} y^{-1} \psi_{i-1}^{-}(y)=-\left(\frac{\mu}{2} \pi_{i+1,0}+\lambda_{1} \pi_{i, 0}\right) y+\mu \pi_{i,-1} \\
i \geq 1
\end{gathered}
$$

3. The recursive relationship for $\varphi_{j}(y), j=0, \pm 1, \pm 2, \ldots$ can be listed as:

$$
\begin{aligned}
& \left(\mu+\lambda_{1} x\right) \varphi_{j+1}(x)-\varphi_{j}(x)+\lambda_{2} \varphi_{j-1}(x)=-\lambda_{1} \pi_{0, j+1} x, \quad j \geq 2, \\
& \left(\mu+\lambda_{1} x\right) \varphi_{2}(x)-\varphi_{1}(x)+\left(\frac{\mu}{2} x^{-1}+\lambda_{2}\right) \varphi_{0}(x)=-\lambda_{1} \pi_{0,2} x+\frac{\mu}{2} \pi_{1,0}, \\
& \left(\mu+\lambda_{1} x\right) \varphi_{1}(x)-\varphi_{0}(x)+\left(\mu+\lambda_{2} x\right) \varphi_{-1}(x)=-\lambda_{1} \pi_{0,1} x-\lambda_{2} \pi_{0,-1} x, \\
& \left(\frac{\mu}{2} x^{-1}+\lambda_{1}\right) \varphi_{0}(x)-\varphi_{-1}(x)+\left(\mu+\lambda_{2} x\right) \varphi_{-2}(x)=-\lambda_{2} \pi_{0,-2} x+\frac{\mu}{2} \pi_{1,0}, \\
& \lambda_{1} \varphi_{j+1}(x)-\varphi_{j}(x)+\left(\mu+\lambda_{2} x\right) \varphi_{j-1}(x)=-\lambda_{2} \pi_{0, j-1} x, \quad j \leq-2 .
\end{aligned}
$$

Proof. Proofs of the recursive relationships for $\psi_{i}^{+}(y), \psi_{i}^{-}(y), i=0,1,2, \ldots$, and $\varphi_{j}(y), j=0, \pm 1, \pm 2, \ldots$, are similar. We only provide details for the proof of the relationship for $\psi_{i}^{+}(y), i=1,2, \ldots$.

In order to proof (2.15), recall (2.1) and (2.2),

$$
\begin{aligned}
& \pi_{i, j}=\lambda_{1} \pi_{i-1, j+1}+\lambda_{2} \pi_{i, j-1}+\mu \pi_{i, j+1}, \quad i \geq 1, \quad j \geq 2, \\
& \pi_{i, 1}=\lambda_{1} \pi_{i-1,2}+\lambda_{2} \pi_{i, 0}+\mu \pi_{i, 2}+\frac{\mu}{2} \pi_{i+1,0}, \quad i \geq 1 .
\end{aligned}
$$


Thus, for $i \geq 1$,

$$
\begin{array}{r}
\psi_{i}^{+}(y)=\sum_{j=1}^{+\infty}\left(\lambda_{1} \pi_{i-1, j+1}+\lambda_{2} \pi_{i, j-1}+\mu \pi_{i, j+1}\right) y^{j}+\frac{\mu}{2} \pi_{j+1,0} y \\
=\lambda_{2} y\left(\psi_{i}^{+}(y)+\pi_{m, 0}\right)+\lambda_{1} y^{-1}\left(\psi_{i-1}^{+}(y)-\pi_{i-1,1} y\right) \\
+\mu y^{-1}\left(\psi_{i}^{+}(y)-\pi_{i, 1} y\right)+\frac{\mu}{2} \pi_{m+1,0} y
\end{array}
$$

Namely,

$$
\left(\mu y^{-1}-1+\lambda_{2} y\right) \psi_{i}^{+}(y)+\lambda_{1} y^{-1} \psi_{i-1}^{+}(y)=-\left(\frac{\mu}{2} \pi_{i+1,0}+\lambda_{2} \pi_{i, 0}\right) y+\mu \pi_{i, 1}
$$

From the recursive relationship for $\psi_{i}^{+}(y)$ and $\psi_{i}^{-}(y), i=0,1,2, \ldots$, the exact tail asymptotic behavior for joint probabilities along the $y$-direction can be derived directly, which will be shown in Chapter 3 . Tail asymptotic properties along the $x$ direction is more challenging, since we cannot directly obtain the tail asymptotics for $\pi_{i, 0}$, which requires a different method (see Chapter 4 ).

After the asymptotic property for the generating function $\varphi_{0}(x)$ and the tail asymptotic property for the boundary probabilities $\pi_{i, 0}$ are obtained, the recursive relationship for $\varphi_{j}(x), j=0, \pm 1, \pm 2, \ldots$, will enables us to carry out the asymptotic analysis for all $\varphi_{j}(x)$, which will lead to a determination of the exact tail asymptotics for the joint probabilities along the $x$-direction. Details will be given in Chapter 4 and Chapter 5.

Another series of important relationships consists of two equations. One is derived for the positive region, and the other one for the negative region. The exact tail analysis for the boundary probabilities along the $x$-direction starts from these two equations using the kernel method.

Lemma 2.2 A set of two equations are listed as: 


$$
\begin{aligned}
-\pi_{\cdot,+}(x, y) h^{+}(x, y)=\varphi_{0}(x) h_{0}^{+}(x, y) & +\varphi_{-1}(x) h_{-1}^{+}(x) \\
& +\pi_{0,0} h_{0,0}^{+}(y)+\pi_{0,-1} h_{0,-1}^{+}(x),
\end{aligned}
$$

and

$$
\begin{aligned}
-\pi_{.,-}(x, y) h^{-}(x, y)=\varphi_{0}(x) h_{0}^{-}(x, y) & +\varphi_{-1}(x) h_{-1}^{-}(x) \\
& +\pi_{0,0} h_{0,0}^{-}(y)+\pi_{0,-1} h_{0,-1}^{-}(x),
\end{aligned}
$$

where

$$
\begin{aligned}
& h^{+}(x, y)=\lambda_{1} x y^{-1}+\lambda_{2} y+\mu y^{-1}-1, h_{0}^{+}(x, y)=\lambda_{2} y+\frac{\mu}{2} x^{-1} y-1, \\
& h_{-1}^{+}(x)=\lambda_{2} x+\mu, h_{0,0}^{+}(y)=\lambda_{2} y+\mu-1, h_{0,-1}^{+}(x)=\lambda_{2} x+\mu ; \\
& h^{-}(x, y)=\lambda_{2} x y^{-1}+\lambda_{1} y+\mu y^{-1}-1, \quad h_{0}^{-}(x, y)=\lambda_{1} y+\frac{\mu}{2} x^{-1} y, \\
& h_{-1}^{-}(x)=-h_{-1}^{+}(x)=-\left(\lambda_{2} x+\mu\right), h_{0,0}^{-}(y)=\lambda_{1} y, \\
& h_{0,-1}^{-}(x)=-h_{0,-1}^{+}(x)=-\left(\lambda_{2} x+\mu\right) .
\end{aligned}
$$

Following Flajolet and Sedgewick [11], we call these functions (2.23) and (2.24) the fundamental forms.

Remark 2.3 It can be noticed that (2.23) and (2.24) are asymmetric. In fact, we put equations in these forms for the advantage that when we add them together, some terms can be cancelled out.

Proof. Proofs of these two fundamental forms are similar, and only the proof of (2.23) is shown as follows: 
Recall that

$$
\begin{aligned}
& \pi_{i, j}=\lambda_{1} \pi_{i-1, j+1}+\lambda_{2} \pi_{i, j-1}+\mu \pi_{i, j+1}, \quad i \geq 1, \quad j \geq 2, \\
& \pi_{i, 1}=\lambda_{1} \pi_{i-1,2}+\lambda_{2} \pi_{i, 0}+\mu \pi_{i, 2}+\frac{\mu}{2} \pi_{i+1,0}, \quad i \geq 1 .
\end{aligned}
$$

Thus,

$$
\begin{aligned}
& \sum_{i \geq 1, j \geq 1} \pi_{i, j} x^{i} y^{j} \\
= & \sum_{i \geq 1, j \geq 1}\left(\lambda_{1} \pi_{i-1, j+1}+\lambda_{2} \pi_{i, j-1}+\mu \pi_{i, j+1}\right) x^{i} y^{j}+\sum_{i \geq 1, j=1}\left(\frac{\mu}{2} \pi_{i+1,0}\right) x^{i} y^{j} .
\end{aligned}
$$

We know

$$
\begin{aligned}
& \sum_{i \geq 1, j \geq 1} \pi_{i-1, j+1} x^{i} y^{j} \\
= & x y^{-1} \sum_{i \geq 0, j \geq 2} \pi_{i, j} x^{i} y^{j} \\
= & x y^{-1}\left(\sum_{i \geq 1, j \geq 1} \pi_{i, j} x^{i} y^{j}+\sum_{i=0, j \geq 1} \pi_{i, j} x^{i} y^{j}-\left(\sum_{i \geq 1, j=1} \pi_{i, j} x^{i} y^{j}+\sum_{i=0, j=1} \pi_{i, j} x^{i} y^{j}\right)\right) \\
= & x y^{-1}\left(\pi_{+,+}(x, y)+\psi_{0}^{+}(y)-\left(\varphi_{1}(x) y+\pi_{0,1} y\right)\right) .
\end{aligned}
$$

Similarly,

$$
\begin{aligned}
\sum_{i \geq 1, j \geq 1} \pi_{i, j-1} x^{i} y^{j} & =y\left(\pi_{+,+}(x, y)+\varphi_{0}(x)\right) \\
\sum_{i \geq 1, j \geq 1} p i_{i, j+1} x^{i} y^{j} & =y^{-1}\left(\pi_{+,+}(x, y)-\varphi_{1}(x) y\right) \\
\sum_{i \geq 1, j \geq 1} \pi_{i+1,0} x^{i} y^{j} & =x^{-1} y\left(\varphi_{0}(x)-\pi_{1,0} x\right) .
\end{aligned}
$$


Thus,

$$
\begin{aligned}
& \left(1-\lambda_{1} x y^{-1}-\lambda_{2} y-\mu y^{-1}\right) \pi_{++}(x, y) \\
= & \psi_{0}^{+}(y) \lambda_{1} x y^{-1}+\varphi_{0}(x)\left(\lambda_{2} y+\frac{\mu}{2} x^{-1} y\right)-\varphi_{1}(x)\left(\lambda_{1} x+\mu\right)-\left(\lambda_{1} x \pi_{0,1}+\frac{\mu}{2} y \pi_{1,0}\right) .
\end{aligned}
$$

Recall (2.14) and (2.20), we have

$$
\begin{array}{r}
0=\left(\mu y^{-1}-1+\lambda_{2} y\right) \psi_{0}^{+}(y)+\left(\frac{\mu}{2} \pi_{1,0}+\lambda_{2} \pi_{0,0}\right) y-\mu \pi_{0,1}, \\
\left(\mu+\lambda_{1} x\right) \varphi_{1}(x)=\varphi_{0}(x)-\left(\mu+\lambda_{2} x\right) \varphi_{-1}(x)-\left(\lambda_{1} \pi_{0,1} x+\lambda_{2} \pi_{0,-1} x\right) .
\end{array}
$$

Also, recall (2.6),

$$
\mu \pi_{0,1}=(1-\mu) \pi_{0,0}-\mu \pi_{0,-1} .
$$

Plus (2.26) and (2.25), replace $\left(\mu+\lambda_{1} x\right) \varphi_{1}(x)$ by using (2.27), and replace $\mu \pi_{0,1}$ by using (2.6), we can get (2.23). 


\section{Chapter 3}

\section{Tail Asymptotic Analysis along the $y$-Direction}

The analysis of exact tail asymptotics along the $y$ direction relies on the analysis of the locations of dominant singularities and local asymptotic properties at the dominant

singularities of the generating function $\psi_{i}^{+}(y)$ and $\psi_{i}^{-}(y), i=1,2, \ldots$ According to the recursive relationships for $\psi_{i}^{+}(y)$ and $\psi_{i}^{-}(y), i=0,1,2, \ldots$, we can find the explicit expressions for the generating functions $\psi_{i}^{+}(y)$ and $\psi_{i}^{-}(y), i=0,1,2, \ldots$, from which the dominant singularities and the behaviors of these unknown generating functions at their dominant singularities are obtained.

\section{Lemma 3.1}

$$
\psi_{i}^{+}(y)=y \sum_{m=0}^{i}\left(-\lambda_{1}\right)^{m} \frac{-\left(\frac{\mu}{2} \pi_{i+1-m, 0}+\lambda_{2} \pi_{i-m, 0}\right) y+\mu \pi_{i-m, 1}}{\left(\lambda_{2} y^{2}-y+\mu\right)^{m+1}}, i=0,1,2, \ldots
$$

is valid on $\mathbb{C}_{y}$, except at a zero of $\lambda_{2} y^{2}-y+\mu$;

$$
\psi_{i}^{-}(y)=y \sum_{m=0}^{i}\left(-\lambda_{2}\right)^{m} \frac{-\left(\frac{\mu}{2} \pi_{i+1-m, 0}+\lambda_{1} \pi_{i-m, 0}\right) y+\mu \pi_{i-m, 1}}{\left(\lambda_{1} y^{2}-y+\mu\right)^{m+1}}, i=0,1,2, \ldots
$$

is valid on $\mathbb{C}_{y}$, expect at a zero of $\lambda_{1} y^{2}-y+\mu$. 
Proof. We can use the mathematical induction to prove this lemma. Proofs of (3.1) and (3.2) are similar, and only the proof of (3.1) is shown as follows. Firstly, $\psi_{0}^{+}(y)$, $|y|<1$, can be written in the form stated in the lemma. Also, assume that for $i=i_{0}$, $|y|<1$, we have

$$
\psi_{i_{0}}^{+}(y)=y \sum_{m=0}^{i_{0}}\left(-\lambda_{1}\right)^{m} \frac{-\left(\frac{\mu}{2} \pi_{i_{0}+1-m, 0}+\lambda_{2} \pi_{i_{0}-m, 0}\right) y+\mu \pi_{i_{0}-m, 1}}{\left(\lambda_{2} y^{2}-y+\mu\right)^{m+1}} .
$$

According to (2.15), we have

$$
\psi_{i_{0}+1}^{+}(y)=\frac{\left(\frac{\mu}{2} \pi_{i_{0}+2,0}+\lambda_{2} \pi_{i_{0}+1,0}\right) y+\mu \pi_{i_{0}+1,1}-\lambda_{1} y^{-1} \psi_{i_{0}}^{+}(y)}{\lambda_{2} y-1+\mu y^{-1}} .
$$

Using (3.3) in (3.4), we get

$$
\psi_{i_{0}+1}^{+}(y)=y \sum_{m=0}^{i_{0}+1}\left(-\lambda_{1}\right)^{m} \frac{-\left(\frac{\mu}{2} \pi_{i_{0}+2-m, 0}+\lambda_{2} \pi_{i_{0}+1-m, 0}\right) y+\mu \pi_{i_{0}+1-m, 1}}{\left(\lambda_{2} y^{2}-y+\mu\right)^{m+1}} .
$$

Obviously, the left hand side of (3.5) is analytic in the whole complex plane except at a zero of $\lambda_{1} y^{2}-y+\mu$, thus $\psi_{i_{0}+1}^{+}(y),|y|<1$, can be at least analytically continued to $\mathbb{C}_{y}$, expect at a zero of $\lambda_{2} y^{2}-y+\mu$.

Lemma 3.1 implies that the zeros of $\lambda_{2} y^{2}-y+\mu$ are possible poles of $\psi_{i}^{+}(y), i=$ $0,1,2, \ldots$, and the the zeros of $\lambda_{1} y^{2}-y+\mu$ are possible poles of $\psi_{i}^{-}(y), i=0,1,2, \ldots$ Let $\lambda_{2} y^{2}-y+\mu=0$, we get $y_{1}^{+}=\frac{1+\sqrt{1-4 \lambda_{2} \mu}}{2 \lambda_{2}}$ and $y_{0}^{+}=\frac{1-\sqrt{1-4 \lambda_{2} \mu}}{2 \lambda_{2}}$. We can see that $y_{1}^{+} \in \mathbb{R}$ and $y_{0}^{+} \in \mathbb{R}$ with $0<y_{0}^{+}<1<y_{1}^{+} . \psi_{i}^{+}(y), i=0,1,2, \ldots$, is a generating function of probabilities, so $\psi_{i}^{+}(y)$ is analytic inside the unit circle. Hence $y_{0}^{+}$is not a pole of it. Therefore, $y_{1}^{+}$is the only pole of $\psi_{i}^{+}(y)$, since $\psi_{i}^{+}(y)$ can be written as $\psi_{i}^{+}(y)=\frac{y \digamma(y)}{\left(y-y_{1}^{+}\right)^{i+1}}$, where $\digamma(y)$ is analytic on the disk $|y| \leq y_{1}^{+}$and it can be shown $\digamma\left(y_{1}^{+}\right) \neq 0$.". Similarly, let $\lambda_{1} y^{2}-y+\mu=0$, we get $y_{1}^{-}=\frac{1+\sqrt{1-4 \lambda_{1} \mu}}{2 \lambda_{1}}$ and $y_{0}^{-}=$ $\frac{1-\sqrt{1-4 \lambda_{1} \mu}}{2 \lambda_{1}}$. Also, $y_{1}^{-} \in \mathbb{R}, y_{0}^{-} \in \mathbb{R}, 0<y_{0}^{-}<1<y_{1}^{-}$, and $y_{1}^{-}$is the dominant 
singularity of $\psi_{i}^{-}(y), i=0,1,2, \ldots$

Lemma 3.2 Local asymptotic behaviors of $\psi_{i}^{-}(y)$ and $\psi_{i}^{-}(y), i=0,1,2, \ldots$, are listed as follows:

$$
\begin{aligned}
& \lim _{y \rightarrow y_{1}^{+}} \psi_{i}^{+}(y)\left(1-\frac{y}{y_{1}^{+}}\right)^{i+1}=c_{i}^{+}\left(y_{1}^{+}\right), \\
& \lim _{y \rightarrow y_{1}^{-}} \psi_{i}^{-}(y)\left(1-\frac{y}{y_{1}^{-}}\right)^{i+1}=c_{i}^{-}\left(y_{1}^{-}\right),
\end{aligned}
$$

where

$$
\begin{aligned}
& c_{i}^{+}\left(y_{1}^{+}\right)=\left(\frac{\lambda_{1}}{\lambda_{2} y_{1}^{+}\left(y_{1}^{+}-y_{0}^{+}\right)}\right)^{i+1} \frac{y_{1}^{+}}{\lambda_{1}}\left(\left(\frac{\mu}{2} \pi_{1,0}+\lambda_{2} \pi_{0,0}\right) y_{1}^{+}-\mu \pi_{0,1}\right), \\
& c_{i}^{-}\left(y_{1}^{-}\right)=\left(\frac{\lambda_{2}}{\lambda_{1} y_{1}^{-}\left(y_{1}^{-}-y_{0}^{-}\right)}\right)^{i+1} \frac{y_{1}^{-}}{\lambda_{2}}\left(\left(\frac{\mu}{2} \pi_{1,0}+\lambda_{2} \pi_{0,0}\right) y_{1}^{-}-\mu \pi_{0,-1}\right) .
\end{aligned}
$$

Remark 3.1 Denote a dominant singularity of a function of $y$ as $y_{d o m}$. All limits are taken in the region $\left\{y:\left|\frac{y}{y_{\text {dom }}}\right| \leq 1+\epsilon,\left|\operatorname{Arg}\left(\frac{y}{y_{\text {dom }}}\right)-1\right| \geq \phi\right\}$, where $\epsilon>0,0<\phi<\pi / 2$, according to the Tauberian-like theorem and the fact that $y_{1}^{+}$(or $y_{1}^{-}$) is the only pole of $\psi_{i}^{+}(y)\left(\right.$ or $\left.\psi_{i}^{-}(y)\right)$.

In this case, $\psi_{i}^{+}(y)$ (or $\psi_{i}^{-}(y)$ ), $i=0,1,2, \ldots$, is analytic on $\mathbb{C}_{y} /\left\{y_{1}^{+}\right\}$(or $\mathbb{C}_{y} /\left\{y_{1}^{-}\right\}$).

Proof. Proofs of (3.6) and (3.7) are similar, and only the proof of (3.6) is provided here. Take the limit in the region $\left\{y:\left|\frac{y}{y_{1}^{+}}\right| \leq 1+\epsilon,\left|\operatorname{Arg}\left(\frac{y}{y_{1}^{+}}\right)-1\right| \geq \phi\right\}$, where $\epsilon>0,0<\phi<\pi / 2$, we have

$$
\begin{aligned}
& \lim _{y \rightarrow y_{1}^{+}}\left(1-\frac{y}{y_{1}^{+}}\right) \frac{1}{\lambda_{2} y^{2}-y+\mu} \\
= & \lim _{y \rightarrow y_{1}^{+}}\left(-\frac{y-y_{1}^{+}}{y_{1}^{+}}\right) \frac{1}{\lambda_{2}\left(y-y_{1}^{+}\right)\left(y-y_{0}^{+}\right)} \\
= & -\frac{1}{\lambda_{2} y_{1}^{+}\left(y_{1}^{+}-y_{0}^{+}\right)} .
\end{aligned}
$$


Thus,

$$
\begin{aligned}
& \lim _{y \rightarrow y_{1}^{+}} \psi_{i}^{+}(y)\left(1-\frac{y}{y_{1}^{+}}\right)^{i+1} \\
= & \lim _{y \rightarrow y_{1}^{+}}\left(\left(1-\frac{y}{y_{1}^{+}}\right) \frac{1}{\lambda_{2} y^{2}-y+\mu}\right)^{i+1} y\left(-\lambda_{1}\right)^{i}\left(-\left(\frac{\mu}{2} \pi_{1,0}+\lambda_{2} \pi_{0,0}\right) y+\mu \pi_{0,1}\right) \\
= & \left(\frac{\lambda_{1}}{\lambda_{2} y_{1}^{+}\left(y_{1}^{+}-y_{0}^{+}\right)}\right)^{i+1} \frac{y_{1}^{+}}{\lambda_{1}}\left(\left(\frac{\mu}{2} \pi_{1,0}+\lambda_{2} \pi_{0,0}\right) y_{1}^{+}-\mu \pi_{0,1}\right) .
\end{aligned}
$$

Lemma 3.3 (geometric decay multiplied by the factor $|n|^{i}$ )

We have for fixed $i, i=0,1, \ldots$, and large $n$,

$$
\pi_{i, n} \sim c_{i}^{+}\left(y_{1}^{+}\right) \frac{n^{i}}{(i+1) !}\left(\frac{1}{y_{1}^{+}}\right)^{n}
$$

and for fixed $i, i=0,1, \ldots$, and large $|n|$ with $n<0$,

$$
\pi_{i, n} \sim c_{i}^{-}\left(y_{1}^{-}\right) \frac{(-n)^{i}}{(i+1) !}\left(\frac{1}{y_{1}^{-}}\right)^{-n}
$$

Remark 3.2 We write $f(x) \sim g(x)$ as $x \rightarrow x_{0}$ to mean $\lim _{x \rightarrow x_{0}} \frac{f(x)}{g(x)}=1$ and the limit is taken over the defined region.

Proof. Proofs of (3.8) and (3.9) are similar, and only the proof of (3.8) is provided here. Notice that $\psi_{i}^{+}(y)$ is analytic in the region $\left\{y:\left|\frac{y}{y_{1}^{+}}\right| \leq 1+\epsilon, \mid \operatorname{Arg}\left(\frac{y}{y_{1}^{+}}\right)-\right.$ $1 \mid \geq \phi\} /\left\{y: \frac{y}{y_{1}^{+}}=1\right\}$, where $\epsilon>0,0<\phi<\pi / 2$. And according to Lemma 3.2, $\psi_{i}^{+}\left(\frac{y}{y_{1}^{+}}\right) \sim c_{i}^{+}\left(y_{1}^{+}\right)\left(1-\frac{y}{y_{1}^{+}}\right)^{-i-1}$ as $\frac{y}{y_{1}^{+}} \rightarrow 1$ in $\left\{y:\left|\frac{y}{y_{1}^{+}}\right|<1+\epsilon,\left|\operatorname{Arg}\left(\frac{y}{y_{1}^{+}}\right)-1\right| \geq \phi\right\}$. Therefore, according to Remark 1.2, $\pi_{i, n} \sim \frac{c_{i}^{+}\left(y_{1}^{+}\right) n^{(i+1)-1}}{\Gamma(i+1)\left(y_{1}^{+}\right)^{n}}$. 


\section{Chapter 4}

\section{Dominant Singularity Analysis of}

$\varphi_{j}(x)$

This chapter focuses on the analysis of dominant singularities of the generating functions $\varphi_{j}(x), j=0, \pm 1, \pm 2, \ldots$, which is the crucial step for using the Tauberian-like theorem. The kernel method is employed here.

\subsection{Kernel method}

The standard kernel method deals with the equation $K(x, y) F(x, y)=A(x, y) G(x)+$ $B(x, y)$, where only $F(x, y)$ and $G(x)$ are unknown functions. The key idea of this method is to find a branch $y=y_{0}(x)$ such that $K\left(x, y_{0}(x)\right)$ is zero. Then for $x \in$ $\left\{x \mid F\left(x, y_{0}(x)\right)<\infty\right\}$, we have $A\left(x, y_{0}(x)\right) G(x)+B\left(x, y_{0}(x)\right)=0$. Thus

$$
G(x)=-B\left(x, y_{0}(x)\right) / A\left(x, y_{0}(x)\right)
$$

for $x \in\left\{x \mid F\left(x, y_{0}(x)\right)<\infty, A\left(x, y_{0}(x)\right) \neq 0\right\} ;$ and

$$
F(x, y)=\frac{-A(x, y) B\left(x, y_{0}(x)\right) / A\left(x, y_{0}(x)\right)+B(x, y)}{K(x, y)}
$$


for $x \in\left\{x \mid F\left(x, y_{0}(x)\right)<\infty, A\left(x, y_{0}(x)\right) \neq 0, K(x, y) \neq 0\right\}$.

This idea has been generalized for random walks in the quarter plane to deal with a fundamental form with two unknown functions on its right hand side. In this thesis, this idea is generalized further by allowing two fundamental forms to be included. By choosing a proper branch in each fundamental form, we obtain relationships between $\varphi_{0}(x)$ and $\varphi_{1}(x)$, according to which explicit expressions of $\varphi_{0}(x)$ and $\varphi_{1}(x)$ can be obtained.

Define $Y_{0}^{+}(x)$ and $Y_{1}^{+}(x)$ as the branches of $y h^{+}(x, y)=0$, with $\left|Y_{0}^{+}(x)\right| \leq\left|Y_{1}^{+}(x)\right|$. $y h^{+}(x, y)=0$, or equivalently $\lambda_{2} y^{2}-y+\left(\lambda_{1} x+\mu\right)=0$, is a kernel equation. Namely,

$$
Y_{0}^{+}(x)=\frac{1-\sqrt{1-4 \lambda_{2}\left(\lambda_{1} x+\mu\right)}}{2 \lambda_{2}}, Y_{1}^{+}(x)=\frac{1+\sqrt{1-4 \lambda_{2}\left(\lambda_{1} x+\mu\right)}}{2 \lambda_{2}}
$$

Similarly, define $Y_{0}^{-}(x)$ and $Y_{1}^{-}(x)$ as the branches of $y h^{-}(x, y)=0$, with $\left|Y_{0}^{-}(x)\right| \leq$ $\left|Y_{1}^{-}(x)\right| \cdot y h^{-}(x, y)=0$, or equivalently $\lambda_{1} y^{2}-y+\left(\lambda_{2} x+\mu\right)=0$, is a kernel equation. Namely,

$$
Y_{0}^{-}(x)=\frac{1-\sqrt{1-4 \lambda_{1}\left(\lambda_{2} x+\mu\right)}}{2 \lambda_{1}}, Y_{1}^{-}(x)=\frac{1+\sqrt{1-4 \lambda_{1}\left(\lambda_{2} x+\mu\right)}}{2 \lambda_{1}} .
$$

Let $x_{1}^{+}=\frac{1-4 \lambda_{2} \mu}{4 \lambda_{1} \lambda_{2}}$. According to (4.1), $x_{1}^{+}$is the branch points of $Y^{+}(x)$. Similarly, let $x_{1}^{-}=\frac{1-4 \lambda_{1} \mu}{4 \lambda_{1} \lambda_{2}}$. According to (4.2), $x_{1}^{-}$is the branch points of $Y^{-}(x)$. Define the following cut planes:

$$
\begin{aligned}
& \widetilde{\mathbb{C}}_{x}^{+}=\mathbb{C}_{x}-\left[x_{1}^{+}, \infty\right), \\
& \widetilde{\mathbb{C}}_{x}^{-}=\mathbb{C}_{x}-\left[x_{1}^{-}, \infty\right) .
\end{aligned}
$$

Lemma 4.1 1. $Y_{0}^{+}(x)\left(Y_{0}^{-}(x)\right)$ is analytic in $\widetilde{\mathbb{C}}_{x}^{+}\left(\widetilde{\mathbb{C}}_{x}^{-}\right)$.

2. There exists $1 \geq \delta>0$, such that $\left|Y_{0}^{+}(x)\right| \leq 1$ whenever $|x|<\delta$. 
3. For $\{x: x \in \mathbb{C},|x|<\delta\}$, we have

$$
0=\varphi_{0}(x) h_{0}^{+}\left(x, Y_{0}^{+}(x)\right)+\varphi_{-1}(x) h_{-1}^{+}(x)+\pi_{0,0} h_{0,0}^{+}\left(Y_{0}^{+}(x)\right)+\pi_{0,-1} h_{0,-1}^{+}(x)
$$

and

$$
0=\varphi_{0}(x) h_{0}^{-}\left(x, Y_{0}^{-}(x)\right)+\varphi_{-1}(x) h_{-1}^{-}(x)+\pi_{0,0} h_{0,0}^{-}\left(Y_{0}^{-}(x)\right)+\pi_{0,-1} h_{0,-1}^{-}(x) .
$$

Proof. Proofs of 3 is obvious and thus omitted here.

Proofs for $Y_{0}^{+}(x)$ and $Y_{0}^{-}(x)$ in 1 are similar, and only the proof for $Y_{0}^{+}(x)$ is detailed here. The functions $Y_{0}^{+}(x)$ is meromorphic in the cut plane $\widetilde{\mathbb{C}}_{x}^{+}$. In addition, $Y_{0}^{+}(x)=$ $\frac{1-\sqrt{1-4 \lambda_{2}\left(\lambda_{1} x+\mu\right)}}{2 \lambda_{2}}=\frac{2\left(\lambda_{1} x+\mu\right)}{1+\sqrt{1-4 \lambda_{2}\left(\lambda_{1} x+\mu\right)}}$, thus $Y_{0}^{+}(x)$ has no poles and $Y_{0}^{+}(x)$ is analytic in $\widetilde{\mathbb{C}}_{x}^{+}$.

Proof of 2 goes as follows: since $Y_{0}^{+}(x)$ is continuous and $Y_{0}^{+}(0)=\frac{1-\sqrt{1-4 \lambda_{2} \mu}}{2 \lambda_{2}}<1$, there exists $1 \geq \delta>0$, such that $\left|Y_{0}^{+}(0)\right| \leq 1$ whenever $|x|<\delta$.

\subsection{Dominant singularity analysis of $\varphi_{j}(x)$}

\section{Lemma 4.21.}

$$
\varphi_{0}(x)=-\frac{\pi_{0,0} x\left(\lambda_{2} Y_{0}^{+}(x)+\lambda_{1} Y_{0}^{-}(x)+\mu-1\right)}{Y_{0}^{+}(x)\left(x \lambda_{2}+\frac{\mu}{2}\right)+Y_{0}^{-}(x)\left(x \lambda_{1}+\frac{\mu}{2}\right)-x}
$$

holds in $\widetilde{\mathbb{C}}_{x}^{+} \cap \widetilde{\mathbb{C}}_{x}^{-} \cap\left\{x: Y_{0}^{+}(x)\left(x \lambda_{2}+\frac{\mu}{2}\right)+Y_{0}^{-}(x)\left(x \lambda_{1}+\frac{\mu}{2}\right)-x \neq 0\right\}$.

2. Let $x_{d o m}$ be a dominant singularity of $\varphi_{0}(x)$, then one of the following two cases must hold:

i) $x_{d o m}=x_{1}^{+}$or $x_{d o m}=x_{1}^{-}$, which is a branch point of $Y^{+}(x)$ or $Y^{-}(x)$;

ii) $x_{\text {dom }}$ is a zero point of $Y_{0}^{+}(x)\left(x \lambda_{2}+\frac{\mu}{2}\right)+Y_{0}^{-}(x)\left(x \lambda_{1}+\frac{\mu}{2}\right)-x$ with $|x|>1$. 
Proof. Plus (4.3) and (4.4), then for $\{x: x \in \mathbb{C},|x|<\delta\}$,

$$
0=\varphi_{0}(x)\left(h_{0}^{+}\left(x, Y_{0}^{+}(x)\right)+h_{0}^{-}\left(x, Y_{0}^{-}(x)\right)\right)+\pi_{0,0}\left(h_{0,0}^{+}\left(Y_{0}^{+}(x)\right)+h_{0,0}^{-}\left(Y_{0}^{-}(x)\right)\right) .
$$

Thus for $\{x: x \in \mathbb{C},|x|<\delta\} \cap\left\{x: x\left(h_{0}^{+}\left(x, Y_{0}^{+}(x)\right)+h_{0}^{-}\left(x, Y_{0}^{-}(x)\right)\right) \neq 0\right\}$,

$$
\varphi_{0}(x)=-\frac{\pi_{0,0} x\left(h_{0,0}^{+}\left(Y_{0}^{+}(x)\right)+h_{0,0}^{-}\left(Y_{0}^{-}(x)\right)\right)}{x\left(h_{0}^{+}\left(x, Y_{0}^{+}(x)\right)+h_{0}^{-}\left(x, Y_{0}^{-}(x)\right)\right)}
$$

Namely, for $\{x: x \in \mathbb{C},|x|<\delta\} \cap\left\{x: Y_{0}^{+}(x)\left(x \lambda_{2}+\frac{\mu}{2}\right)+Y_{0}^{-}(x)\left(x \lambda_{1}+\frac{\mu}{2}\right)-x \neq 0\right\}$,

$$
\varphi_{0}(x)=-\frac{\pi_{0,0} x\left(\lambda_{2} Y_{0}^{+}(x)+\lambda_{1} Y_{0}^{-}(x)+\mu-1\right)}{Y_{0}^{+}(x)\left(x \lambda_{2}+\frac{\mu}{2}\right)+Y_{0}^{-}(x)\left(x \lambda_{1}+\frac{\mu}{2}\right)-x} .
$$

The right hand side of (4.6) is analytic in $\widetilde{\mathbb{C}}_{x}^{+} \cap \widetilde{\mathbb{C}}_{x}^{-} \cap\left\{x: Y_{0}^{+}(x)\left(x \lambda_{2}+\frac{\mu}{2}\right)+Y_{0}^{-}(x)\left(x \lambda_{1}+\right.\right.$ $\left.\left.\frac{\mu}{2}\right)-x \neq 0\right\}$, thus $\varphi_{0}(x),\{x: x \in \mathbb{C},|x|<\delta\}$, can be analytically continued at least to $\widetilde{\mathbb{C}}_{x}^{+} \cap \widetilde{\mathbb{C}}_{x}^{-} \cap\left\{x: Y_{0}^{+}(x)\left(x \lambda_{2}+\frac{\mu}{2}\right)+Y_{0}^{-}(x)\left(x \lambda_{1}+\frac{\mu}{2}\right)-x \neq 0\right\}$. Therefore (4.6) holds for $\widetilde{\mathbb{C}}_{x}^{+} \cap \widetilde{\mathbb{C}}_{x}^{-} \cap\left\{x: Y_{0}^{+}(x)\left(x \lambda_{2}+\frac{\mu}{2}\right)+Y_{0}^{-}(x)\left(x \lambda_{1}+\frac{\mu}{2}\right)-x \neq 0\right\}$, which leads to a proof of 2 .

Lemma 4.3 1. For $x \in \mathbb{C}_{x}$, we have

$$
\varphi_{j}(x)=\frac{2 \lambda_{2}\left(\pi_{0, j-1}+\varphi_{j-1}(x)\right)}{1+\sqrt{1-4 \lambda_{2}\left(\lambda_{1} x+\mu\right)}}-\pi_{0, j}, \quad j \geq 2
$$

holds except at a pole (if there is any) of $\varphi_{j-1}(x)$;

$$
\varphi_{1}(x)=\frac{2 \lambda_{2} x \pi_{0,0}+\left(2 \lambda_{2} x+\mu\right) \varphi_{0}(x)}{x\left(1+\sqrt{1-4 \lambda_{2}\left(\lambda_{1} x+\mu\right)}\right)}-\pi_{0,1}
$$

holds except at a pole (if there is any) of $\varphi_{0}(x)$;

$$
\varphi_{-1}(x)=\frac{2 \lambda_{1} x \pi_{0,0}+\left(2 \lambda_{1} x+\mu\right) \varphi_{0}(x)}{x\left(1+\sqrt{1-4 \lambda_{1}\left(\lambda_{2} x+\mu\right)}\right)}-\pi_{0,-1}
$$


holds except at a pole (if there is any) of $\varphi_{0}(x)$;

$$
\varphi_{j}(x)=\frac{2 \lambda_{1}\left(\pi_{0, j+1}+\varphi_{j+1}(x)\right)}{1+\sqrt{1-4 \lambda_{1}\left(\lambda_{2} x+\mu\right)}}-\pi_{0, j}, \quad j \leq-2
$$

holds except at a pole (if there is any) of $\varphi_{j+1}(x)$;

2. The dominant singularity of $\varphi_{j}(x), j= \pm 1, \pm 2, \pm 3, \ldots$, is the dominant singularity of $\varphi_{0}(x)$.

Proof. Firstly, we show (4.8) and (4.9).

For a proof of (4.9), from (4.3), we have for $\{x: x \in \mathbb{C},|x|<\delta\}$,

$$
\varphi_{-1}(x) h_{-1}^{+}(x)=-\varphi_{0}(x) h_{0}^{+}\left(x, Y_{0}^{+}(x)\right)-\pi_{0,0} h_{0,0}^{+}\left(Y_{0}^{+}(x)\right)-\pi_{0,-1} h_{0,-1}^{+}(x)
$$

The right hand side of (4.11) is analytic in $\mathbb{C}_{x}^{+}$except at a pole (if there is any) of $\varphi_{0}(x)$. Using (4.5) in (4.11), we have for $\{x: x \in \mathbb{C},|x|<\delta\}$,

$$
\begin{gathered}
h_{-1}^{+}(x) \varphi_{-1}(x)=\frac{\pi_{0,0}\left(h_{0,0}^{+}\left(Y_{0}^{+}(x)\right)+h_{0,0}^{-}\left(Y_{0}^{-}(x)\right)\right)}{x\left(h_{0}^{+}\left(x, Y_{0}^{+}(x)\right)+h_{0}^{-}\left(x, Y_{0}^{-}(x)\right)\right)} x h_{0}^{+}\left(x, Y_{0}^{+}(x)\right) \\
-\pi_{0,0} h_{0,0}^{+}\left(Y_{0}^{+}(x)\right)-\pi_{0,-1} h_{0,-1}^{+}(x) .
\end{gathered}
$$

Namely,

$$
\begin{gathered}
\left(\mu+\lambda_{2} x\right) \varphi_{-1}(x)=\frac{\pi_{0,0} x\left(\lambda_{2} Y_{0}^{+}(x)+\lambda_{1} Y_{0}^{-}(x)+\mu-1\right)\left(\left(\lambda_{2}+\frac{\mu}{2 x}\right) Y_{0}^{+}(x)-1\right)}{Y_{0}^{+}(x)\left(x \lambda_{2}+\frac{\mu}{2}\right)+Y_{0}^{-}(x)\left(x \lambda_{1}+\frac{\mu}{2}\right)-x} \\
-\pi_{0,0}\left(\lambda_{2} Y_{0}^{+}(x)+\mu-1\right)-\pi_{0,-1}\left(\lambda_{2} x+\mu\right) .
\end{gathered}
$$


Also,

$$
\begin{aligned}
&\left(\lambda_{2} x+\mu\right) \varphi_{-1}(x) \\
&= \frac{\pi_{0,0}\left(\lambda_{2} Y_{0}^{+}(x)+\lambda_{1} Y_{0}^{-}(x)+\mu-1\right)\left(\left(\lambda_{2} x+\frac{\mu}{2}\right) Y_{0}^{+}(x)-x\right)}{Y_{0}^{+}(x)\left(x \lambda_{2}+\frac{\mu}{2}\right)+Y_{0}^{-}(x)\left(x \lambda_{1}+\frac{\mu}{2}\right)-x} \\
&-\pi_{0,0}\left(\lambda_{2} Y_{0}^{+}(x)+\mu-1\right)-\pi_{0,-1}\left(\lambda_{2} x+\mu\right) \\
&= \frac{\pi_{0,0}\left(\lambda_{2} Y_{0}^{+}(x)+\lambda_{1} Y_{0}^{-}(x)+\mu-1\right)\left(\left(\lambda_{2} x+\frac{\mu}{2}\right) Y_{0}^{+}(x)-x+Y_{0}^{-}(x)\left(x \lambda_{1}+\frac{\mu}{2}\right)\right)}{Y_{0}^{+}(x)\left(x \lambda_{2}+\frac{\mu}{2}\right)+Y_{0}^{-}(x)\left(x \lambda_{1}+\frac{\mu}{2}\right)-x} \\
&-\frac{\pi_{0,0}\left(\lambda_{2} Y_{0}^{+}(x)+\lambda_{1} Y_{0}^{-}(x)+\mu-1\right) Y_{0}^{-}(x)\left(x \lambda_{1}+\frac{\mu}{2}\right)}{Y_{0}^{+}(x)\left(x \lambda_{2}+\frac{\mu}{2}\right)+Y_{0}^{-}(x)\left(x \lambda_{1}+\frac{\mu}{2}\right)-x} \\
&-\pi_{0,0}\left(\lambda_{2} Y_{0}^{+}(x)+\mu-1\right)-\pi_{0,-1}\left(\lambda_{2} x+\mu\right) \\
&=\lambda_{1} \pi_{0,0} Y_{0}^{-}(x)-\frac{\pi_{0,0}\left(\lambda_{2} Y_{0}^{+}(x)+\lambda_{1} Y_{0}^{-}(x)+\mu-1\right) Y_{0}^{-}(x)\left(x \lambda_{1}+\frac{\mu}{2}\right)}{Y_{0}^{+}(x)\left(x \lambda_{2}+\frac{\mu}{2}\right)+Y_{0}^{-}(x)\left(x \lambda_{1}+\frac{\mu}{2}\right)-x}-\pi_{0,-1}\left(\lambda_{2} x+\mu\right) \\
&=\lambda_{1} \pi_{0,0} Y_{0}^{-}(x)+x^{-1}\left(\frac{\mu}{2}+\lambda_{1} x\right) \varphi_{0}(x) Y_{0}^{-}(x)-\pi_{0,-1}\left(\lambda_{2} x+\mu\right) .
\end{aligned}
$$

We know

$$
Y_{0}^{-}(x)=\frac{1-\sqrt{1-4 \lambda_{1}\left(\lambda_{2} x+\mu\right)}}{2 \lambda_{1}}=\frac{2\left(\lambda_{2} x+\mu\right)}{\left.1+\sqrt{1-4 \lambda_{1}\left(\lambda_{2} x+\mu\right)}\right)}
$$

Thus, we have for $\{x: x \in \mathbb{C},|x|<\delta\}$,

$$
\varphi_{-1}(x)=\frac{2 \lambda_{1} x \pi_{0,0}+\left(2 \lambda_{1} x+\mu\right) \varphi_{0}(x)}{x\left(1+\sqrt{1-4 \lambda_{1}\left(\lambda_{2} x+\mu\right)}\right)}-\pi_{0,-1}
$$

The right hand side of (4.13) is analytic in $\mathbb{C}_{x}$ except at a pole (if there is any) of $\varphi_{0}(x)$. Therefore, (4.13) holds in $\mathbb{C}_{x}$ except at a pole (if there is any) of $\varphi_{0}(x)$.

For the proof of (4.8), according to $(2.20)$, we have for $\{x: x \in \mathbb{C},|x|<\delta\}$,

$$
\left(\mu+\lambda_{1} x\right) \varphi_{1}(x)=-\lambda_{1} \pi_{0,1} x-\lambda_{2} \pi_{0,-1} x+\varphi_{0}(x)-\left(\mu+\lambda_{2} x\right) \varphi_{-1}(x)
$$


Input (4.12) and (4.6) into (4.14), we have for $\{x: x \in \mathbb{C},|x|<\delta\}$,

$$
\begin{aligned}
& \left(\mu+\lambda_{1} x\right) \varphi_{1}(x) \\
& =-\lambda_{1} \pi_{0,1} x-\lambda_{2} \pi_{0,-1} x-\frac{\pi_{0,0} x\left(\lambda_{2} Y_{0}^{+}(x)+\lambda_{1} Y_{0}^{-}(x)+\mu-1\right)}{Y_{0}^{+}(x)\left(x \lambda_{2}+\frac{\mu}{2}\right)+Y_{0}^{-}(x)\left(x \lambda_{1}+\frac{\mu}{2}\right)-x} \\
& -\frac{\pi_{0,0} x\left(\lambda_{2} Y_{0}^{+}(x)+\lambda_{1} Y_{0}^{-}(x)+\mu-1\right)\left(\left(\lambda_{2}+\frac{\mu}{2 x}\right) Y_{0}^{+}(x)-1\right)}{Y_{0}^{+}(x)\left(x \lambda_{2}+\frac{\mu}{2}\right)+Y_{0}^{-}(x)\left(x \lambda_{1}+\frac{\mu}{2}\right)-x} \\
& +\pi_{0,0}\left(\lambda_{2} Y_{0}^{+}(x)+\mu-1\right)+\pi_{0,-1}\left(\lambda_{2} x+\mu\right) \\
& =-\lambda_{1} \pi_{0,1} x+\pi_{0,0}\left(\lambda_{2} Y_{0}^{+}(x)+\mu-1\right)+\pi_{0,-1} \mu \\
& -\frac{\pi_{0,0} x\left(\lambda_{2} Y_{0}^{+}(x)+\lambda_{1} Y_{0}^{-}(x)+\mu-1\right)\left(\lambda_{2}+\frac{\mu}{2 x}\right) Y_{0}^{+}(x)}{Y_{0}^{+}(x)\left(x \lambda_{2}+\frac{\mu}{2}\right)+Y_{0}^{-}(x)\left(x \lambda_{1}+\frac{\mu}{2}\right)-x} \\
& =-\lambda_{1} \pi_{0,1} x+\pi_{0,0}(\mu-1)+\pi_{0,-1} \mu+\pi_{0,0} \lambda_{2} Y_{0}^{+}(x)+x^{-1} \varphi_{0}(x)\left(\lambda_{2} x+\frac{\mu}{2}\right) Y_{0}^{+}(x) \\
& =-\left(\lambda_{1} x+\mu\right) \pi_{0,1}+\left(\pi_{0,0} \lambda_{2}+x^{-1} \varphi_{0}(x)\left(\lambda_{2} x+\frac{\mu}{2}\right)\right) Y_{0}^{+}(x) \text {. }
\end{aligned}
$$

We know

$$
Y_{0}^{+}(x)=\frac{1-\sqrt{1-4 \lambda_{2}\left(\lambda_{1} x+\mu\right)}}{2 \lambda_{2}}=\frac{2\left(\lambda_{1} x+\mu\right)}{1+\sqrt{1-4 \lambda_{2}\left(\lambda_{1} x+\mu\right)}} .
$$

Thus, for $\{x: x \in \mathbb{C},|x|<\delta\}$,

$$
\varphi_{1}(x)=\frac{2 \lambda_{2} x \pi_{0,0}+\left(2 \lambda_{2} x+\mu\right) \varphi_{0}(x)}{x\left(1+\sqrt{1-4 \lambda_{2}\left(\lambda_{1} x+\mu\right)}\right)}-\pi_{0,1}
$$

The right hand side of (4.15) is analytic in $\mathbb{C}_{x}$ except at a pole (if there is any) of $\varphi_{0}(x)$. Therefore, (4.15) holds in $\mathbb{C}_{x}$ except at a pole (if there is any) of $\varphi_{0}(x)$.

Secondly, we show (4.7) and (4.10). Since the proof of (4.7) is similar to that of (4.10), we provide details for (4.10). We show (4.10) for $j=-2$ first. Then, using the same procedure, we can show that if for $j=k, k \leq-2,\{x: x \in \mathbb{C},|x|<\delta\}$, $\varphi_{k}(x)=\frac{2 \lambda_{1}\left(\pi_{0, k+1}+\varphi_{k+1}(x)\right)}{1+\sqrt{1-4 \lambda_{1}\left(\lambda_{2} x+\mu\right)}}-\pi_{0, k}$, then for $j=k-1,\{x: x \in \mathbb{C},|x|<\delta\}, \varphi_{k-1}(x)=$ 
$\frac{2 \lambda_{1}\left(\pi_{0, k}+\varphi_{k}(x)\right)}{1+\sqrt{1-4 \lambda_{1}\left(\lambda_{2} x+\mu\right)}}-\pi_{0, k-1}$. Therefore, for $j \leq-2,\{x: x \in \mathbb{C},|x|<\delta\}$, we have

$$
\varphi_{j}(x)=\frac{2 \lambda_{1}\left(\pi_{0, j+1}+\varphi_{j+1}(x)\right)}{1+\sqrt{1-4 \lambda_{1}\left(\lambda_{2} x+\mu\right)}}-\pi_{0, j}
$$

The right hand side of $(4.16)$ is analytic in $\mathbb{C}_{x}$ except at a pole (if there is any) of $\varphi_{j+1}(x)$. Therefore, (4.16) holds in $\mathbb{C}_{x}$ except at a pole (if there is any) of $\varphi_{j+1}(x)$. The proof of (4.10) for $j=-2$ is provided as follows:

According to (2.21), we have for $\{x: x \in \mathbb{C},|x|<\delta\}$,

$$
x\left(\mu+\lambda_{2} x\right) \varphi_{-2}(x)=-\left(\frac{\mu}{2}+\lambda_{1} x\right) \varphi_{0}(x)+x \varphi_{-1}(x)+\left(-\lambda_{2} \pi_{0,-2} x+\frac{\mu}{2} \pi_{1,0}\right) x .
$$

Also, from (4.13), we have for $\{x: x \in \mathbb{C},|x|<\delta\}$,

$$
2\left(\frac{\mu}{2}+\lambda_{1} x\right) \varphi_{0}(x)=x\left(\varphi_{-1}(x)+\pi_{0,-1}\right)\left(1+\sqrt{1-4 \lambda_{1}\left(\lambda_{2} x+\mu\right)}\right)-2 \pi_{0,0} \lambda_{1} x
$$

Using (4.18) in (4.17), we have for $\{x: x \in \mathbb{C},|x|<\delta\}$,

$$
\begin{aligned}
& x\left(\mu+\lambda_{2} x\right) \varphi_{-2}(x) \\
=- & x\left(\varphi_{-1}(x)+\pi_{0,-1}\right) \frac{1+\sqrt{1-4 \lambda_{1}\left(\lambda_{2} x+\mu\right)}}{2}+\pi_{0,0} \lambda_{1} x \\
& +x \varphi_{-1}(x)+\left(-\lambda_{2} \pi_{0,-2} x+\frac{\mu}{2} \pi_{1,0}\right) x \\
=- & x\left(\varphi_{-1}(x)+\pi_{0,-1}\right) \frac{1+\sqrt{1-4 \lambda_{1}\left(\lambda_{2} x+\mu\right)}}{2}+\pi_{0,0} \lambda_{1} x \\
& +x \varphi_{-1}(x)+\left(-\lambda_{2} \pi_{0,-2} x+\left(\pi_{0,-1}-\lambda_{1} \pi_{0,0}-\mu \pi_{0,-2}\right)\right) x \\
= & x\left(\varphi_{-1}(x)+\pi_{0,-1}\right) \frac{1-\sqrt{1-4 \lambda_{1}\left(\lambda_{2} x+\mu\right)}}{2}-\left(\lambda_{2} x+\mu\right) \pi_{0,-2} x .
\end{aligned}
$$


Thus, for $\{x: x \in \mathbb{C},|x|<\delta\}$,

$$
\varphi_{-2}(x)=\frac{2 \lambda_{1}\left(\pi_{0,-1}+\varphi_{-1}(x)\right)}{1+\sqrt{1-4 \lambda_{1}\left(\lambda_{2} x+\mu\right)}}-\pi_{0,-2}
$$

The right hand side of (4.19) is analytic in $\mathbb{C}_{x}$ except at a pole (if there is any) of $\varphi_{-1}(x)$. Therefore, (4.19) holds in $\mathbb{C}_{x}$ except at a pole (if there is any) of $\varphi_{-1}(x)$. Thus we complete the proof.

For convenience, define $H(x)=x\left(h_{0}^{+}\left(x, Y_{0}^{+}(x)\right)+h_{0}^{-}\left(x, Y_{0}^{-}(x)\right)\right)$.

Lemma 4.4 $x=1, x=\left(\frac{\mu}{\lambda_{1}+\lambda_{2}}\right)^{2}$ and $x=-\frac{\mu\left(2\left(\lambda_{1}+\lambda_{2}\right)+\mu\left(\lambda_{1}-\lambda_{2}\right)^{2}\right)}{4 \lambda_{1} \lambda_{2}}$ are the only possible zeros of $H(x)$.

Proof. We can show that $x=1, x=\left(\frac{\mu}{\lambda_{1}+\lambda_{2}}\right)^{2}, x=-\frac{\mu\left(2\left(\lambda_{1}+\lambda_{2}\right)+\mu\left(\lambda_{1}-\lambda_{2}\right)^{2}\right)}{4 \lambda_{1} \lambda_{2}}$ are the only possible zeros of $H(x) H^{*}(x)$, where

$$
H^{*}(x)=x\left(h_{0}^{+}\left(x, Y_{1}^{+}(x)\right)+h_{0}^{-}\left(x, Y_{1}^{-}(x)\right)\right)
$$

We have

$$
\begin{aligned}
H(x) & =\left(x \lambda_{2}+\frac{\mu}{2}\right) Y_{0}^{+}(x)+\left(x \lambda_{1}+\frac{\mu}{2}\right) Y_{0}^{-}(x)-x \\
H^{*}(x) & =\left(x \lambda_{2}+\frac{\mu}{2}\right) Y_{1}^{+}(x)+\left(x \lambda_{1}+\frac{\mu}{2}\right) Y_{1}^{-}(x)-x
\end{aligned}
$$

Thus,

$$
\begin{aligned}
& H(x) H^{*}(x) \\
= & \left(x \lambda_{1}+\frac{\mu}{2}\right)\left(x \lambda_{2}+\frac{\mu}{2}\right)\left(Y_{0}^{+}(x) Y_{1}^{-}(x)+Y_{0}^{-}(x) Y_{1}^{+}(x)\right) \\
& +\left(x \lambda_{2}+\frac{\mu}{2}\right)^{2} Y_{0}^{+}(x) Y_{1}^{+}(x)+\left(x \lambda_{1}+\frac{\mu}{2}\right)^{2} Y_{0}^{-}(x) Y_{1}^{-}(x) \\
& -x\left(x \lambda_{2}+\frac{\mu}{2}\right)\left(Y_{0}^{+}(x)+Y_{1}^{+}(x)\right)+\left(x \lambda_{1}+\frac{\mu}{2}\right)\left(Y_{0}^{-}(x)+Y_{1}^{-}(x)\right)+x^{2} .
\end{aligned}
$$


Also, we have

$$
\begin{aligned}
& Y_{0}^{+}(x)+Y_{1}^{+}(x)=\frac{1}{\lambda_{2}}, \quad Y_{0}^{-}(x)+Y_{1}^{-}(x)=\frac{1}{\lambda_{1}} \\
& Y_{0}^{+}(x) Y_{1}^{+}(x)=\frac{\lambda_{1} x+\mu}{\lambda_{2}}, \quad Y_{0}^{-}(x) Y_{1}^{-}(x)=\frac{\lambda_{2} x+\mu}{\lambda_{1}} ; \\
& Y_{0}^{+}(x) Y_{1}^{-}(x)+Y_{0}^{-}(x) Y_{1}^{+}(x)=\frac{1-\sqrt{1-4 \lambda_{2}\left(\lambda_{1} x+\mu\right)} \sqrt{1-4 \lambda_{1}\left(\lambda_{2} x+\mu\right)}}{2 \lambda_{1} \lambda_{2}}
\end{aligned}
$$

Therefore,

$$
\begin{aligned}
& H(x) H^{*}(x) \\
= & \left(x \lambda_{1}+\frac{\mu}{2}\right)\left(x \lambda_{2}+\frac{\mu}{2}\right) \frac{1}{2 \lambda_{1} \lambda_{2}}\left(1-\sqrt{1-4 \lambda_{2}\left(\lambda_{1} x+\mu\right)} \sqrt{1-4 \lambda_{1}\left(\lambda_{2} x+\mu\right)}\right) \\
& +\left(x \lambda_{2}+\frac{\mu}{2}\right)^{2} \frac{\lambda_{1} x+\mu}{\lambda_{2}}+\left(x \lambda_{1}+\frac{\mu}{2}\right)^{2} \frac{\lambda_{2} x+\mu}{\lambda_{1}} \\
& -x\left(\left(x \lambda_{2}+\frac{\mu}{2}\right) \frac{1}{\lambda_{2}}+\left(x \lambda_{1}+\frac{\mu}{2}\right) \frac{1}{\lambda_{1}}\right)+x^{2} .
\end{aligned}
$$

Thus, if $H(x) H^{*}(x)=0$, we have

$$
\begin{gathered}
4 \lambda_{1}^{2} \lambda_{2}^{2}\left(\left(x \lambda_{1}+\frac{\mu}{2}\right)\left(x \lambda_{2}+\frac{\mu}{2}\right) \frac{1}{2 \lambda_{1} \lambda_{2}}+\left(x \lambda_{2}+\frac{\mu}{2}\right)^{2} \frac{\lambda_{1} x+\mu}{\lambda_{2}}\right. \\
\left.+\left(x \lambda_{1}+\frac{\mu}{2}\right)^{2} \frac{\lambda_{2} x+\mu}{\lambda_{1}}-x\left(\left(x \lambda_{2}+\frac{\mu}{2}\right) \frac{1}{\lambda_{2}}+\left(x \lambda_{1}+\frac{\mu}{2}\right) \frac{1}{\lambda_{1}}\right)+x^{2}\right)^{2} \\
=4 \lambda_{1}^{2} \lambda_{2}^{2}\left(\left(x \lambda_{1}+\frac{\mu}{2}\right)\left(x \lambda_{2}+\frac{\mu}{2}\right) \frac{1}{2 \lambda_{1} \lambda_{2}} \sqrt{1-4 \lambda_{2}\left(\lambda_{1} x+\mu\right)} \sqrt{1-4 \lambda_{1}\left(\lambda_{2} x+\mu\right)}\right)^{2}
\end{gathered}
$$

which is equivalent to

$$
\begin{aligned}
0= & \left(\frac{\mu^{2}}{2}\left(\lambda_{1}-\lambda_{2}\right)\left(\left(\lambda_{1}+\lambda_{2}\right) x+\mu\right)\right)^{2}-\frac{\mu^{2}}{2} x\left(2 \lambda_{1} \lambda_{2} x+\mu\left(\lambda_{1}+\lambda_{2}\right)\right) \\
& -4\left(\lambda_{1} \lambda_{2} x^{2}+\frac{\mu}{2}\left(\lambda_{1}+\lambda_{2}\right) x+\frac{\mu^{2}}{4}\right) \frac{\mu^{2}}{4}\left(\lambda_{1}-\lambda_{2}\right)^{2} x \\
& +\mu^{2}\left(2 \lambda_{1} \lambda_{2} x+\mu\left(\lambda_{1}+\lambda_{2}\right)\right)\left(\left(\lambda_{1}^{2}+\lambda_{2}^{2}\right) x^{2}+\frac{\mu^{2}}{2}+\mu\left(\lambda_{1}+\lambda_{2}\right) x\right)
\end{aligned}
$$


or

$$
0=4 \lambda_{1} \lambda_{2}\left(\lambda_{1}+\lambda_{2}\right)^{2}(x-1)\left(x-\left(\frac{\mu}{\lambda_{1}+\lambda_{2}}\right)^{2}\right)\left(x+\frac{\mu\left(2\left(\lambda_{1}+\lambda_{2}\right)+\mu\left(\lambda_{1}-\lambda_{2}\right)^{2}\right)}{4 \lambda_{1} \lambda_{2}}\right) .
$$

Thus, the proof is complete.

Lemma $4.5\left(\frac{\mu}{\lambda_{1}+\lambda_{2}}\right)^{2}$ is the only possible zero of $H(x)$ with the modulus greater than 1 , and $\left(\frac{\mu}{\lambda_{1}+\lambda_{2}}\right)^{2}$ is a zero of $H(x)$ if and only if $\max \left\{\lambda_{1}, \lambda_{2}\right\} \leq \frac{1-\mu}{2 \mu}$.

Proof. It is easy to verify that:

(i) $H\left(\left(\frac{\mu}{\lambda_{1}+\lambda_{2}}\right)^{2}\right)=0$ if and only if $\max \left\{\lambda_{1}, \lambda_{2}\right\} \leq \frac{1-\mu}{2 \mu}$;

(ii) $H\left(-\frac{\mu\left[2\left(\lambda_{1}+\lambda_{2}\right)+\mu\left(\lambda_{1}-\lambda_{2}\right)^{2}\right]}{4 \lambda_{1} \lambda_{2}}\right) \neq 0$, and $H^{*}\left(-\frac{\mu\left[2\left(\lambda_{1}+\lambda_{2}\right)+\mu\left(\lambda_{1}-\lambda_{2}\right)^{2}\right]}{4 \lambda_{1} \lambda_{2}}\right)=0$.

Denote $x_{*}=\left(\frac{\mu}{\lambda_{1}+\lambda_{2}}\right)^{2}$, and recall $x_{1}^{+}=\frac{1-4 \lambda_{2} \mu}{4 \lambda_{1} \lambda_{2}}$ and $x_{1}^{-}=\frac{1-4 \lambda_{1} \mu}{4 \lambda_{1} \lambda_{2}}$.

\section{Lemma 4.6}

$$
x_{d o m}= \begin{cases}x_{1}^{-}<x_{1}^{+}, & \text {if } \lambda_{1}>\frac{1-\mu}{2 \mu}, \lambda_{1}>\lambda_{2}, \\ x_{1}^{+}<x_{1}^{-}, & \text {if } \lambda_{2}>\frac{1-\mu}{2 \mu}, \lambda_{1}<\lambda_{2}, \\ x_{*}<\min \left\{x_{1}^{+}, x_{1}^{-}\right\}, & \text {if } \max \left\{\lambda_{1}, \lambda_{2}\right\}<\frac{1-\mu}{2 \mu}, \\ x_{*}=x_{1}^{-}<x_{1}^{+}, & \text {if } \lambda_{2}<\lambda_{1}=\frac{1-\mu}{2 \mu}, \\ x_{*}=x_{1}^{+}<x_{1}^{-}, & \text {if } \lambda_{1}<\lambda_{2}=\frac{1-\mu}{2 \mu} .\end{cases}
$$

Proof. Recall $x_{1}^{+}=\frac{1-4 \lambda_{2} \mu}{4 \lambda_{1} \lambda_{2}}$, and $x_{2}^{+}=\infty$ are the branch points of $Y^{+}(x)$; and $x_{1}^{-}=\frac{1-4 \lambda_{1} \mu}{4 \lambda_{1} \lambda_{2}}$ and $x_{2}^{-}=\infty$ are branch points of $Y^{-}(x)$.

It is easy to have the following results:

(i) $x_{1}^{+}>1$ and $x_{1}^{-}>1$, since $x_{1}^{+}-1=\frac{\left(2 \lambda_{2}-1\right)^{2}}{4 \lambda_{1} \lambda_{2}}$ and $x_{1}^{-}-1=\frac{\left(2 \lambda_{1}-1\right)^{2}}{4 \lambda_{1} \lambda_{2}}$.

(ii) $x_{1}^{+}=x_{1}^{-}$, if $\lambda_{1}=\lambda_{2} ; x_{1}^{+}<x_{1}^{-}$, if $\lambda_{1}<\lambda_{2} ; x_{1}^{+}>x_{1}^{-}$, if $\lambda_{1}>\lambda_{2}$, since $x_{1}^{+}-x_{1}^{-}=$ $\frac{\left(\lambda_{1}-\lambda_{2}\right) \mu}{\lambda_{1} \lambda_{2}}$. 
(iii) $x_{*} \leq x_{1}^{+}$, and the equality holds if and only if $\lambda_{2}=\frac{1-\mu}{2 \mu}$. It is true since

$$
x_{*}-x_{1}^{+}=-\frac{\left(2 \lambda_{2} \mu-(1-\mu)\right)^{2}}{4 \lambda_{1} \lambda_{2}\left(\lambda_{1}+\lambda_{2}\right)^{2}} \leq 0
$$

and the equality holds if and only if $\lambda_{2}=\frac{1-\mu}{2 \mu}$.

(iv) $x_{*} \leq x_{1}^{-}$, and the equality holds if and only if $\lambda_{1}=\frac{1-\mu}{2 \mu}$. It is true since

$$
x_{*}-x_{1}^{-}=-\frac{\left(2 \lambda_{1} \mu-(1-\mu)\right)^{2}}{4 \lambda_{1} \lambda_{2}\left(\lambda_{1}+\lambda_{2}\right)^{2}} \leq 0
$$

and the equality holds if and only if $\lambda_{1}=\frac{1-\mu}{2 \mu}$.

(v) If $\lambda_{1}=\lambda_{2}$, then $x_{\text {dom }}=x_{*}<\min \left\{x_{1}^{+}, x_{1}^{-}\right\}$. It is true since $\lambda_{1}=\lambda_{2} \geq \frac{1-\mu}{2 \mu}$ is impossible, otherwise, $\lambda_{1}+\lambda_{2} \geq \frac{1-\mu}{\mu}>1-\mu$, which conflicts $\lambda_{1}+\lambda_{2}=1-\mu$. 


\section{Chapter 5}

\section{Tail Asymptotic Analysis along the $x$-Direction}

In this chapter, the behavior of $\varphi_{j}(x), j=0, \pm 1, \pm 2, \ldots$, at the dominant singularity is detailed and the tail asymptotic results along the $x$-direction are given.

\subsection{Asymptotic analysis of $\varphi_{0}(x)$}

Rewrite

$$
\begin{aligned}
Y_{0}^{+}(x) & =p^{+}+q^{+} \sqrt{1-\frac{x}{x_{1}^{+}}}, \quad Y_{0}^{-}(x)=p^{-}+q^{-} \sqrt{1-\frac{x}{x_{1}^{-}}} \\
Y_{0}^{+}\left(x_{1}^{+}\right)-Y_{0}^{+}(x) & =-q^{+} \sqrt{1-\frac{x}{x_{1}^{+}}}, \quad Y_{0}^{-}\left(x_{1}^{-}\right)-Y_{0}^{-}(x)=-q^{-} \sqrt{1-\frac{x}{x_{1}^{-}}}, \\
H(x) & =p+q_{1}(x) \sqrt{1-\frac{x}{x_{1}^{+}}}+q_{2}(x) \sqrt{1-\frac{x}{x_{1}^{-}}},
\end{aligned}
$$


where $p^{+}, q^{+}, p^{-}, q^{-}$and $p$ are constants; $q_{1}(x)$ and $q_{2}(x)$ are analytic in the region $\widetilde{\mathbb{C}}_{x}^{+} \cap \widetilde{\mathbb{C}}_{x}^{-}$.

$$
\begin{aligned}
p^{+} & =\frac{1}{2 \lambda_{2}}, \quad q^{+}=-\sqrt{\frac{\lambda_{1} x_{1}^{+}}{\lambda_{2}}}, \quad p^{-}=\frac{1}{2 \lambda_{1}}, \quad q^{-}=-\sqrt{\frac{\lambda_{2} x_{1}^{-}}{\lambda_{1}}} \\
p & =\frac{\mu}{4}\left(\frac{1}{\lambda_{1}}+\frac{1}{\lambda_{2}}\right), q_{1}(x)=-\sqrt{\frac{\lambda_{1} x_{1}^{+}}{\lambda_{2}}}\left(\lambda_{2} x+\frac{\mu}{2}\right), q_{2}(x)=-\sqrt{\frac{\lambda_{2} x_{1}^{-}}{\lambda_{1}}}\left(\lambda_{1} x+\frac{\mu}{2}\right) .
\end{aligned}
$$

Define

$$
T\left(x, Y_{0}^{+}(x), Y_{0}^{-}(x)\right) \triangleq-\frac{\pi_{0,0} x\left(\lambda_{2} Y_{0}^{+}(x)+\lambda_{1} Y_{0}^{-}(x)+\mu-1\right)}{Y_{0}^{+}(x)\left(x \lambda_{2}+\frac{\mu}{2}\right)+Y_{0}^{-}(x)\left(x \lambda_{1}+\frac{\mu}{2}\right)-x} .
$$

Let $x_{d o m}$ be the dominant singularity of $\varphi_{0}(x)$, or the singular point of $\varphi_{0}(x)$ with the smallest modulus. Clearly, $x_{d o m}=x_{*}$, or $x_{d o m}=x_{1}^{+}$, or $x_{d o m}=x_{1}^{-}$.

The following Lemma shows the behavior of $\varphi_{0}(x)$ at $x_{d o m}$.

Lemma 5.1 For the function $\varphi_{0}(x)$, a total of three types of asymptotics exist as $x$ approaches to the dominant singularity of $\varphi_{0}(x)$ based on the detailed property of the dominant singularity.

Case 1: If $x_{\text {dom }}=x_{*}<\min \left\{x_{1}^{+}, x_{1}^{-}\right\}$, then

$$
\lim _{x \rightarrow x_{d o m}}\left(1-\frac{x}{x_{d o m}}\right) \varphi_{0}(x)=c_{0,1}\left(x_{d o m}\right)
$$

where

$$
c_{0,1}\left(x_{d o m}\right)=\frac{\pi_{0,0}\left(\lambda_{2} Y_{0}^{+}\left(x_{d o m}\right)+\lambda_{1} Y_{0}^{-}\left(x_{d o m}\right)+\mu-1\right)}{\left.\frac{d}{d x}\left[q_{1}(x) \sqrt{1-\frac{x}{x_{1}^{+}}}+q_{2}(x) \sqrt{1-\frac{x}{x_{1}^{-}}}\right]\right|_{x=x_{d o m}}} .
$$

Case 2: If $x_{d o m}=x_{*}=x_{1}^{+}<x_{1}^{-}$, or $x_{d o m}=x_{*}=x_{1}^{-}<x_{1}^{+}$, then

$$
\lim _{x \rightarrow x_{d o m}} \sqrt{1-x / x_{d o m}} \varphi_{0}(x)=c_{0,2}\left(x_{d o m}\right)
$$


where

$$
c_{0,2}\left(x_{d o m}\right)=\left\{\begin{array}{cl}
-\frac{\pi_{0,0} x_{d o m}\left(\lambda_{1} Y_{0}^{-}\left(x_{d o m}\right)+\mu-1\right)}{q_{1}\left(x_{d o m}\right)}, & \text { if } x_{d o m}=x_{*}=x_{1}^{+}<x_{1}^{-}, \\
-\frac{\pi_{0,0} x_{d o m}\left(\lambda_{2} Y_{0}^{+}\left(x_{d o m}\right)+\mu-1\right)}{q_{2}\left(x_{d o m}\right)}, & \text { if } x_{d o m}=x_{*}=x_{1}^{-}<x_{1}^{+} .
\end{array}\right.
$$

Case 3: If $x_{d o m}=x_{1}^{+}<x_{1}^{-}$, or $x_{d o m}=x_{1}^{-}<x_{1}^{+}$, then

$$
\lim _{x \rightarrow x_{d o m}} \sqrt{1-x / x_{d o m}} \varphi_{0}^{\prime}(x)=c_{0,3}\left(x_{d o m}\right)
$$

where

$$
c_{0,3}\left(x_{d o m}\right)= \begin{cases}-\left.\frac{q^{+}}{2 x_{1}^{+}} \frac{\partial T}{\partial Y_{0}^{+}(x)}\right|_{\left(x_{d o m}, Y_{0}^{+}\left(x_{d o m}\right), Y_{0}^{-}\left(x_{d o m}\right)\right)}, & \text { if } x_{d o m}=x_{*}=x_{1}^{+}<x_{1}^{-}, \\ -\left.\frac{q^{-}}{2 x_{1}^{-}} \frac{\partial T}{\partial Y_{0}^{-}(x)}\right|_{\left(x_{d o m}, Y_{0}^{+}\left(x_{d o m}\right), Y_{0}^{-}\left(x_{d o m}\right)\right)}, & \text { if } x_{d o m}=x_{*}=x_{1}^{-}<x_{1}^{+} .\end{cases}
$$

Proof. Recall for $x \in \widetilde{\mathbb{C}}_{x}^{+} \cap \widetilde{\mathbb{C}}_{x}^{-} \cap\left\{x: h_{0}^{+}\left(x, Y_{0}^{+}(x)\right)+h_{0}^{-}\left(x, Y_{0}^{-}(x)\right) \neq 0\right\}$,

$$
\varphi_{0}(x)=-\frac{\pi_{0,0} x\left(\lambda_{2} Y_{0}^{+}(x)+\lambda_{1} Y_{0}^{-}(x)+\mu-1\right)}{H(x)} .
$$

Case 1: If $x_{d o m}=x_{*}<\min \left\{x_{1}^{+}, x_{1}^{-}\right\}$, then

$$
H(x)=H(x)-H\left(x_{*}\right)=q^{*}(x)\left(1-\frac{x}{x_{*}}\right)
$$

where

$$
\begin{aligned}
q^{*}(x)=-x_{*}\left(\frac{q_{1}(x) \sqrt{1-\frac{x}{x_{1}^{+}}}-q_{1}\left(x_{*}\right) \sqrt{1-\frac{x_{*}^{*}}{x_{1}^{+}}}}{x-x_{*}}\right. \\
\left.+\frac{q_{2}(x) \sqrt{1-\frac{x}{x_{1}^{-}}}-q_{2}\left(x_{*}\right) \sqrt{1-\frac{x_{*}}{x_{1}^{-}}}}{x-x_{*}}\right) .
\end{aligned}
$$


Thus,

$$
\left(1-\frac{x}{x_{d o m}}\right) \varphi_{0}(x)=-\frac{\pi_{0,0} x\left(\lambda_{2} Y_{0}^{+}(x)+\lambda_{1} Y_{0}^{-}(x)+\mu-1\right)}{q^{*}(x)}
$$

Therefore,

$$
\begin{aligned}
& \lim _{x \rightarrow x_{d o m}}\left(1-\frac{x}{x_{d o m}}\right) \varphi_{0}(x) \\
= & \lim _{x \rightarrow x_{d o m}}-\frac{\pi_{0,0} x\left(\lambda_{2} Y_{0}^{+}(x)+\lambda_{1} Y_{0}^{-}(x)+\mu-1\right)}{q^{*}} \\
= & \frac{\pi_{0,0}\left(\lambda_{2} Y_{0}^{+}\left(x_{d o m}\right)+\lambda_{1} Y_{0}^{-}\left(x_{d o m}\right)+\mu-1\right)}{\left.\frac{d}{d x}\left[q_{1}(x) \sqrt{1-\frac{x}{x_{1}^{+}}}+q_{2}(x) \sqrt{1-\frac{x}{x_{1}^{-}}}\right]\right|_{x=x_{d o m}}} .
\end{aligned}
$$

Case 2: We provide details for $x_{d o m}=x_{*}=x_{1}^{+}<x_{1}^{-}$. Other cases can be similarly proved.

If $x_{d o m}=x_{*}=x_{1}^{+}<x_{1}^{-}$, then $H\left(x_{1}^{+}\right)=0$.

$$
\begin{aligned}
H(x)=H(x)-H\left(x_{1}^{+}\right) & =q_{1}(x) \sqrt{1-\frac{x}{x_{1}^{+}}}+q_{2}(x) \sqrt{1-\frac{x}{x_{1}^{-}}}-q_{2}\left(x_{1}^{+}\right) \sqrt{1-\frac{x_{1}^{+}}{x_{1}^{-}}} \\
& =q_{1}(x) \sqrt{1-\frac{x}{x_{1}^{+}}}+q_{2}^{*}(x)\left(1-\frac{x}{x_{1}^{+}}\right),
\end{aligned}
$$

where

$$
q_{2}^{*}(x)=-x_{1}^{+} \frac{q_{2}(x) \sqrt{1-\frac{x}{x_{1}^{-}}}-q_{2}\left(x_{1}^{+}\right) \sqrt{1-\frac{x_{1}^{+}}{x_{1}^{-}}}}{x-x_{1}^{+}}
$$

is analytic at $x=x_{1}^{+}$.

Thus,

$$
\sqrt{1-\frac{x}{x_{d o m}}} \varphi_{0}(x)=-\frac{\pi_{0,0} x\left(\lambda_{1} Y_{0}^{-}(x)+\mu-1\right)}{q_{1}(x)+q_{2}^{*}(x) \sqrt{1-\frac{x}{x_{1}^{+}}}} .
$$


Therefore,

$$
\lim _{x \rightarrow x_{d o m}} \sqrt{1-x / x_{d o m}} \varphi_{0}(x)=-\frac{\pi_{0,0} x_{d o m}\left(\lambda_{1} Y_{0}^{-}\left(x_{d o m}\right)+\mu-1\right)}{q_{1}\left(x_{d o m}\right)} .
$$

Case 3: We provide details for $x_{d o m}=x_{1}^{+}<x_{1}^{-}$. Other cases can be similarly proved.

Recall

$$
T\left(x, Y_{0}^{+}(x), Y_{0}^{-}(x)\right) \triangleq-\frac{\pi_{0,0} x\left(\lambda_{2} Y_{0}^{+}(x)+\lambda_{1} Y_{0}^{-}(x)+\mu-1\right)}{Y_{0}^{+}(x)\left(x \lambda_{2}+\frac{\mu}{2}\right)+Y_{0}^{-}(x)\left(x \lambda_{1}+\frac{\mu}{2}\right)-x} .
$$

Thus,

$$
\frac{d T\left(x, Y_{0}^{+}(x), Y_{0}^{-}(x)\right)}{d x}=\frac{\partial T}{\partial x}+\frac{\partial T}{\partial Y_{0}^{+}(x)} \frac{d Y_{0}^{+}(x)}{d x}+\frac{\partial T}{\partial Y_{0}^{-}(x)} \frac{d Y_{0}^{-}(x)}{d x}
$$

Also,

$$
\begin{aligned}
& \frac{d Y_{0}^{+}(x)}{d x}=q^{+} \frac{d \sqrt{1-\frac{x}{x_{1}^{+}}}}{d x}=-\frac{q^{+}}{2 x_{1}^{+} \sqrt{1-\frac{x}{x_{1}^{+}}}}, \\
& \frac{d Y_{0}^{+}(x)}{d x}=-\frac{q^{-}}{2 x_{1}^{-} \sqrt{1-\frac{x}{x_{1}^{-}}}}, \\
& \lim _{x \rightarrow x_{d o m}} \sqrt{1-x / x_{d o m}} \frac{\partial T}{\partial x}=0, \\
& \lim _{x \rightarrow x_{d o m}} \sqrt{1-x / x_{d o m}} \frac{\partial T}{\partial Y_{0}^{-}(x)} \frac{d Y_{0}^{-}(x)}{d x}=0 .
\end{aligned}
$$


Therefore,

$$
\begin{aligned}
& \lim _{x \rightarrow x_{d o m}} \sqrt{1-x / x_{d o m}} \varphi_{0}^{\prime}(x) \\
& =\lim _{x \rightarrow x_{d o m}} \sqrt{1-x / x_{d o m}} \frac{\partial T}{\partial Y_{0}^{+}(x)} \frac{d Y_{0}^{+}(x)}{d x} \\
& =-\left.\frac{q^{+}}{2 x_{1}^{+}} \frac{\partial T}{\partial Y_{0}^{+}(x)}\right|_{\left(x_{d o m}, Y_{0}^{+}\left(x_{d o m}\right), Y_{0}^{-}\left(x_{d o m}\right)\right)}
\end{aligned}
$$

Remark 5.1 For random walks in the quarter plane, at most four types of exact tail asymptotics are obtained: exact geometric, geometric multiplied by a prefactor of $n^{-\frac{1}{2}}$, geometric multiplied by a prefactor of $n^{-\frac{3}{2}}$, and geometric multiplied by a prefactor of $n$. The fourth type is missing in our case, since $x_{1}^{+}=x_{1}^{-}<x_{*}$ is required for that type, while if $\lambda_{1}=\lambda_{2}$, then $x_{*}<x_{1}^{+}=x_{1}^{-}$.

\subsection{Asymptotic analysis of $\varphi_{j}(x), j \neq 0$}

Lemma 5.2 For $x \in \mathbb{C}_{x}, j= \pm 1, \pm 2, \ldots$, we have

$$
\varphi_{j}(x)=\left\{\begin{array}{c}
\sum_{k=0}^{j-1}\left(\frac{2 \lambda_{2}}{1+\sqrt{1-4 \lambda_{2}\left(\lambda_{1} x+\mu\right)}}\right)^{k}\left(\frac{2 \lambda_{2} \pi_{0, j-k-1}}{1+\sqrt{1-4 \lambda_{2}\left(\lambda_{1} x+\mu\right)}}-\pi_{0, j-k}\right) \\
+\left(\frac{2 \lambda_{2}}{1+\sqrt{1-4 \lambda_{2}\left(\lambda_{1} x+\mu\right)}}\right)^{j-1} \frac{2 \lambda_{2}+\mu x^{-1}}{1+\sqrt{1-4 \lambda_{2}\left(\lambda_{1} x+\mu\right)}} \varphi_{0}(x), \\
\text { if } j \geq 1, \\
\sum_{k=0}^{-j-1}\left(\frac{2 \lambda_{1}}{1+\sqrt{1-4 \lambda_{1}\left(\lambda_{2} x+\mu\right)}}\right)^{k}\left(\frac{2 \lambda_{1} \pi_{0, j-k+1}}{1+\sqrt{1-4 \lambda_{1}\left(\lambda_{2} x+\mu\right)}}-\pi_{0, j-k}\right) \\
+\left(\frac{2 \lambda_{1}+\mu x^{-1}}{1+\sqrt{1-4 \lambda_{1}\left(\lambda_{2} x+\mu\right)}}\right)^{-j-1} \\
\frac{\text { if } j \leq-1 .}{1+\sqrt{1-4 \lambda_{1}\left(\lambda_{2} x+\mu\right)}} \varphi_{0}(x),
\end{array}\right.
$$


Lemma 5.3 Case 1: If $x_{\text {dom }}=x_{*}<\min \left\{x_{1}^{+}, x_{1}^{-}\right\}$, then

$$
\lim _{x \rightarrow x_{d o m}}\left(1-\frac{x}{x_{d o m}}\right) \varphi_{j}(x)=c_{j, 1}\left(x_{d o m}\right)
$$

where

$$
c_{j, 1}\left(x_{d o m}\right)=\left\{\begin{array}{c}
\left(\frac{2 \lambda_{2}}{1+\sqrt{1-4 \lambda_{2}\left(\lambda_{1} x_{d o m}+\mu\right)}}\right)^{j-1}\left(\frac{2 \lambda_{2}+\mu x_{d o m}^{-1}}{1+\sqrt{1-4 \lambda_{2}\left(\lambda_{1} x_{d o m}+\mu\right)}}\right) c_{0,1}\left(x_{d o m}\right), \\
\text { if } j \geq 1, \\
\left(\frac{2 \lambda_{1}}{1+\sqrt{1-4 \lambda_{1}\left(\lambda_{2} x_{d o m}+\mu\right)}}\right)^{-j-1}\left(\frac{2 \lambda_{1}+\mu x_{d o m}^{-1}}{1+\sqrt{1-4 \lambda_{1}\left(\lambda_{2} x_{d o m}+\mu\right)}}\right) c_{0,1}\left(x_{d o m}\right), \\
\text { if } j \leq-1 .
\end{array}\right.
$$

Case 2: If $x_{d o m}=x_{*}=x_{1}^{+}<x_{1}^{-}$, or $x_{d o m}=x_{*}=x_{1}^{-}<x_{1}^{+}$, then

$$
\lim _{x \rightarrow x_{d o m}} \sqrt{1-x / x_{d o m}} \varphi_{j}(x)=c_{j, 2}\left(x_{d o m}\right)
$$

where

(a) under $x_{d o m}=x_{*}=x_{1}^{+}<x_{1}^{-}$,

$$
c_{j, 2}\left(x_{d o m}\right)= \begin{cases}\left(2 \lambda_{2}\right)^{j-1}\left(2 \lambda_{2}+\mu x_{d o m}^{-1}\right) c_{0,2}\left(x_{d o m}\right), & \text { if } j \geq 1, \\ \left(\frac{2 \lambda_{1}}{1+\sqrt{1-4 \lambda_{1}\left(\lambda_{2} x_{d o m}+\mu\right)}}\right)^{-j-1}\left(\frac{2 \lambda_{1}+\mu x_{d o m}^{-1}}{1+\sqrt{1-4 \lambda_{1}\left(\lambda_{2} x_{d o m}+\mu\right)}}\right) c_{0,2}\left(x_{d o m}\right), & \text { if } j \leq-1 ;\end{cases}
$$


(b) under $x_{d o m}=x_{*}=x_{1}^{-}<x_{1}^{+}$,

$$
c_{j, 2}\left(x_{d o m}\right)=\left\{\begin{array}{lc}
\left(\frac{2 \lambda_{2}}{1+\sqrt{1-4 \lambda_{2}\left(\lambda_{1} x_{d o m}+\mu\right)}}\right)^{j-1}\left(\frac{2 \lambda_{2}+\mu x_{d o m}^{-1}}{1+\sqrt{1-4 \lambda_{2}\left(\lambda_{1} x_{d o m}+\mu\right)}}\right) c_{0,2}\left(x_{d o m}\right), \\
& \text { if } j \geq 1, \\
\left(2 \lambda_{1}\right)^{-j-1}\left(2 \lambda_{1}+\mu x_{d o m}^{-1}\right) c_{0,2}\left(x_{d o m}\right), & \text { if } j \leq-1 .
\end{array}\right.
$$

Case 3: If $x_{d o m}=x_{1}^{+}<x_{1}^{-}$, or $x_{d o m}=x_{1}^{-}<x_{1}^{+}$, then

$$
\lim _{x \rightarrow x_{d o m}} \sqrt{1-x / x_{d o m}} \varphi_{j}^{\prime}(x)=c_{j, 3}\left(x_{d o m}\right)
$$

where

(a) under $x_{d o m}=x_{1}^{+}<x_{1}^{-}$,

$$
c_{j, 3}\left(x_{d o m}\right)=\left\{\begin{array}{c}
\left(2 \lambda_{2}\right)^{j-1}\left(2 \lambda_{2}+\mu x_{d o m}^{-1}\right) c_{0,3}\left(x_{d o m}\right)+\frac{2 \lambda_{1} \lambda_{2}}{1-4 \lambda_{2} \mu} \sum_{k=0}^{j-1}\left(2 \lambda_{2}\right)^{k} \varphi_{j-k}\left(x_{d o m}\right), \\
\text { if } j \geq 1, \\
\left(\frac{2 \lambda_{1}}{1+\sqrt{1-4 \lambda_{1}\left(\lambda_{2} x_{d o m}+\mu\right)}}\right)^{-j-1}\left(\frac{2 \lambda_{1}+\mu x_{d o m}^{-1}}{1+\sqrt{1-4 \lambda_{1}\left(\lambda_{2} x_{d o m}+\mu\right)}}\right) c_{0,3}\left(x_{d o m}\right), \\
\text { if } j \leq-1 ;
\end{array}\right.
$$

(b) under $x_{d o m}=x_{1}^{-}<x_{1}^{+}$,

$$
c_{j, 3}\left(x_{d o m}\right)=\left\{\begin{array}{c}
\left(\frac{2 \lambda_{2}}{1+\sqrt{1-4 \lambda_{2}\left(\lambda_{1} x_{d o m}+\mu\right)}}\right)^{j-1}\left(\frac{2 \lambda_{2}+\mu x_{d o m}^{-1}}{\left.1+\sqrt{1-4 \lambda_{2}\left(\lambda_{1} x_{d o m}+\mu\right.}\right)}\right) c_{0,3}\left(x_{d o m}\right), \\
\text { if } j \geq 1, \\
\left(2 \lambda_{1}\right)^{j-1}\left(2 \lambda_{1}+\mu x_{d o m}^{-1}\right) c_{0,3}\left(x_{d o m}\right)+\frac{2 \lambda_{1} \lambda_{2}}{\sqrt{1-4 \lambda_{1} \mu}} \sum_{k=0}^{-j-1}\left(2 \lambda_{1}\right)^{k} \varphi_{j-k}\left(x_{d o m}\right), \\
\text { if } j \leq-1 .
\end{array}\right.
$$


Proof. Case 1 and 2: It can be easily concluded according to (4.7), (4.8), (4.9), (4.10) and (5.1) respectively.

Case 3: According to (4.7), (4.8), (4.9) and (4.10), we have

$$
\varphi_{j}^{\prime}(x)= \begin{cases}\frac{2 \lambda_{2} \varphi_{j-1}^{\prime}(x)+\frac{2 \lambda_{1} \lambda_{2} \varphi_{j}(x)}{\sqrt{1-4 \lambda_{2}\left(\lambda_{1} x+\mu\right)}}}{1+\sqrt{1-4 \lambda_{2}\left(\lambda_{1} x+\mu\right)}}, & \text { if } j \geq 2, \\ \frac{\left(2 \lambda_{2}+\mu x^{-1}\right) \varphi_{0}^{\prime}(x)-\left(\frac{\mu}{x^{2}}\right) \varphi_{0}(x)+\frac{2 \lambda_{1} \lambda_{2} \varphi_{1}(x)}{\sqrt{1-4 \lambda_{2}\left(\lambda_{1} x+\mu\right)}}}{1+\sqrt{1-4 \lambda_{2}\left(\lambda_{1} x+\mu\right)}}, & \text { if } j=1, \\ \frac{\left(2 \lambda_{1}+\mu x^{-1}\right) \varphi_{0}^{\prime}(x)-\left(\frac{\mu}{x^{2}}\right) \varphi_{0}(x)+\frac{2 \lambda_{1} \lambda_{2} \varphi_{-1}(x)}{\sqrt{1-4 \lambda_{1}\left(\lambda_{2} x+\mu\right)}}}{1+\sqrt{1-4 \lambda_{1}\left(\lambda_{2} x+\mu\right)}}, & \text { if } j=-1, \\ \frac{2 \lambda_{1} \varphi_{j+1}^{\prime}(x)+\frac{2 \lambda_{1} \lambda_{2} \varphi_{j}(x)}{\sqrt{1-4 \lambda_{1}\left(\lambda_{2} x+\mu\right)}}}{1+\sqrt{1-4 \lambda_{1}\left(\lambda_{2} x+\mu\right)}}, & \text { if } j \leq-2 .\end{cases}
$$

Also,

$$
\begin{aligned}
& \lim _{x \rightarrow x_{1}^{+}} \sqrt{1-x / x_{1}^{+}} \frac{1}{\sqrt{1-4 \lambda_{2}\left(\lambda_{1} x+\mu\right)}}=\frac{1}{\sqrt{4 \lambda_{1} \lambda_{2} x_{1}^{+}}}=\frac{1}{1-4 \lambda_{2} \mu} \\
& \lim _{x \rightarrow x_{1}^{-}} \sqrt{1-x / x_{1}^{-}} \frac{1}{\sqrt{1-4 \lambda_{1}\left(\lambda_{2} x+\mu\right)}}=\frac{1}{\sqrt{4 \lambda_{1} \lambda_{2} x_{1}^{-}}}=\frac{1}{1-4 \lambda_{1} \mu} .
\end{aligned}
$$

Therefore, under $x_{d o m}=x_{1}^{+}<x_{1}^{-}$, we have

$$
\begin{aligned}
c_{j, 3}\left(x_{d o m}\right) & =2 \lambda_{2} c_{j-1,3}\left(x_{d o m}\right)+\frac{2 \lambda_{1} \lambda_{2}}{\sqrt{1-4 \lambda_{2} \mu}} \varphi_{j}\left(x_{d o m}\right), \quad j \geq 2, \\
c_{1,3}\left(x_{d o m}\right) & =\left(2 \lambda_{2}+\mu x_{d o m}^{-1}\right) c_{0,3}\left(x_{d o m}\right)+\frac{2 \lambda_{1} \lambda_{2}}{\sqrt{1-4 \lambda_{2} \mu}} \varphi_{1}\left(x_{d o m}\right), \\
c_{-1,3}\left(x_{d o m}\right) & =\frac{2 \lambda_{1}+\mu x_{d o m}^{-1}}{1+\sqrt{1-4 \lambda_{1}\left(\lambda_{2} x_{d o m}+\mu\right)}} c_{0,3}\left(x_{d o m}\right), \\
c_{j, 3}\left(x_{d o m}\right) & =\frac{2 \lambda_{1}}{1+\sqrt{1-4 \lambda_{1}\left(\lambda_{2} x_{d o m}+\mu\right)}} c_{j+1,3}\left(x_{d o m}\right), \quad j \leq-2 .
\end{aligned}
$$


Thus,

$$
c_{j, 3}\left(x_{d o m}\right)=\left\{\begin{array}{c}
\left(2 \lambda_{2}\right)^{j-1}\left(2 \lambda_{2}+\mu x_{d o m}^{-1}\right) c_{0,3}\left(x_{d o m}\right)+\frac{2 \lambda_{1} \lambda_{2}}{1-4 \lambda_{2} \mu} \sum_{k=0}^{j-1}\left(2 \lambda_{2}\right)^{k} \varphi_{j-k}\left(x_{d o m}\right), \\
\text { if } j \geq 1, \\
\left(\frac{2 \lambda_{1}}{1+\sqrt{1-4 \lambda_{1}\left(\lambda_{2} x_{d o m}+\mu\right)}}\right)^{-j-1}\left(\frac{2 \lambda_{1}+\mu x_{d o m}^{-1}}{1+\sqrt{1-4 \lambda_{1}\left(\lambda_{2} x_{d o m}+\mu\right)}}\right) c_{0,3}\left(x_{d o m}\right), \\
\text { if } j \leq-1 .
\end{array}\right.
$$

Similarly, under $x_{d o m}=x_{1}^{-}<x_{1}^{+}$, we have

$$
\begin{aligned}
c_{j, 3}\left(x_{d o m}\right) & =\frac{2 \lambda_{2}}{1+\sqrt{1-4 \lambda_{2}\left(\lambda_{1} x_{d o m}+\mu\right)}} c_{j-1,3}\left(x_{d o m}\right), \quad j \geq 2, \\
c_{1,3}\left(x_{d o m}\right) & =\frac{2 \lambda_{2}+\mu x_{d o m}^{-1}}{1+\sqrt{1-4 \lambda_{2}\left(\lambda_{1} x_{d o m}+\mu\right)}} c_{0,3}\left(x_{d o m}\right), \\
c_{-1,3}\left(x_{d o m}\right) & =\left(2 \lambda_{1}+\mu x_{d o m}^{-1}\right) c_{0,3}\left(x_{d o m}\right)+\frac{2 \lambda_{1} \lambda_{2}}{\sqrt{1-4 \lambda_{1} \mu}} \varphi_{-1}\left(x_{d o m}\right), \\
c_{j, 3}\left(x_{d o m}\right) & =2 \lambda_{1} c_{j+1,3}\left(x_{d o m}\right)+\frac{2 \lambda_{1} \lambda_{2}}{\sqrt{1-4 \lambda_{1} \mu}} \varphi_{j}\left(x_{d o m}\right), \quad j \leq-2 .
\end{aligned}
$$

Thus,

$$
c_{j, 3}\left(x_{d o m}\right)=\left\{\begin{array}{cc}
\left(\frac{2 \lambda_{2}}{1+\sqrt{1-4 \lambda_{2}\left(\lambda_{1} x_{d o m}+\mu\right)}}\right)^{j-1}\left(\frac{2 \lambda_{2}+\mu x_{d o m}^{-1}}{1+\sqrt{1-4 \lambda_{2}\left(\lambda_{1} x_{d o m}+\mu\right)}}\right) c_{0,3}\left(x_{d o m}\right), \\
\text { if } j \geq 1, \\
\left(2 \lambda_{1}\right)^{j-1}\left(2 \lambda_{1}+\mu x_{d o m}^{-1}\right) c_{0,3}\left(x_{d o m}\right)+\frac{2 \lambda_{1} \lambda_{2}}{\sqrt{1-4 \lambda_{1} \mu}} \sum_{k=0}^{-j-1}\left(2 \lambda_{1}\right)^{k} \varphi_{j-k}\left(x_{d o m}\right), \\
\text { if } j \leq-1 .
\end{array}\right.
$$




\subsection{Tail asymptotic results}

Lemma 5.4 Case 1: (exact geometric decay): For $x_{d o m}=x_{*}<\min \left\{x_{1}^{+}, x_{1}^{-}\right\}$, namely $\max \left\{\lambda_{1}, \lambda_{2}\right\}<\frac{1-\mu}{2 \mu}$, we have for fixed $j, j=0, \pm 1, \pm 2, \ldots$, and large $n$,

$$
\pi_{n, j} \sim c_{j, 1}\left(x_{d o m}\right)\left(\frac{1}{x_{d o m}}\right)^{n+1}
$$

Case 2: (geometric decay multiplied by the factor $n^{-1 / 2}$ ): For $x_{d o m}=x_{*}=x_{1}^{+}<x_{1}^{-}$, namely $\lambda_{1}<\lambda_{2}=\frac{1-\mu}{2 \mu}$; or $x_{d o m}=x_{*}=x_{1}^{-}<x_{1}^{+}$, namely, $\lambda_{2}<\lambda_{1}=\frac{1-\mu}{2 \mu}$, we have for fixed $j, j=0, \pm 1, \pm 2, \ldots$, and large $n$,

$$
\pi_{n, j} \sim \frac{c_{j, 2}\left(x_{\text {dom }}\right)}{\sqrt{\pi}} n^{-1 / 2}\left(\frac{1}{x_{d o m}}\right)^{n+1}
$$

Case 3: (geometric decay multiplied by the factor $n^{-3 / 2}$ ): For $x_{d o m}=x_{1}^{+}<x_{1}^{-}$, namely, $\lambda_{2}>\frac{1-\mu}{2 \mu}, \lambda_{1}<\lambda_{2}$; or $x_{\text {dom }}=x_{1}^{-}<x_{1}^{+}$, namely, $\lambda_{1}>\frac{1-\mu}{2 \mu}, \lambda_{1}>\lambda_{2}$, we have for fixed $j, j=0, \pm 1, \pm 2, \ldots$, and large $n$,

$$
\pi_{n, j} \sim \frac{c_{j, 3}\left(x_{d o m}\right)}{\sqrt{\pi}} n^{-3 / 2}\left(\frac{1}{x_{d o m}}\right)^{n}
$$

Remark 5.2 All limits are taken in the region $\left\{x:\left|\frac{x}{x_{\text {dom }}}\right| \leq 1+\epsilon,\left|\operatorname{Arg}\left(\frac{x}{x_{\text {dom }}}\right)-1\right| \geq\right.$ $\phi\}$, where $\epsilon>0,0<\phi<\pi / 2$.

Proof. Notice that $\varphi_{j}$ is analytic in the region $\Delta /\left\{x_{\text {dom }}\right\}$, namely $\left\{x:\left|\frac{x}{x_{\text {dom }}}\right| \leq\right.$ $\left.1+\epsilon,\left|\operatorname{Arg}\left(\frac{x}{x_{\text {dom }}}\right)-1\right| \geq \phi\right\} /\left\{x: \frac{x}{x_{\text {dom }}}=1\right\}$, where $\epsilon>0,0<\phi<\pi / 2$.

Case 1: According to Lemma 5.1 and Lemma 5.3, $j=0, \pm 1, \pm 2, \ldots$,

$$
\varphi_{j}\left(\frac{x}{x_{d o m}}\right) \sim c_{j, 1}\left(x_{d o m}\right)\left(1-\frac{x}{x_{d o m}}\right)^{-1} \text { as } \frac{x}{x_{d o m}} \rightarrow 1 \text { in } \triangle .
$$


Thus, from Remark 1.2,

$$
\pi_{n, j} \sim \frac{c_{j, 1}\left(x_{d o m}\right)}{\Gamma(1)\left(x_{d o m}\right)^{n+1}} n^{1-1}=c_{j, 1}\left(x_{d o m}\right)\left(\frac{1}{x_{d o m}}\right)^{n+1}
$$

Case 2: According to Lemma 5.1 and Lemma $5.3, j=0, \pm 1, \pm 2, \ldots$,

$$
\varphi_{j}\left(\frac{x}{x_{d o m}}\right) \sim c_{j, 2}\left(x_{d o m}\right)\left(1-\frac{x}{x_{d o m}}\right)^{-1 / 2} \text { as } \frac{x}{x_{d o m}} \rightarrow 1 \text { in } \triangle
$$

Thus, from Remark 1.2,

$$
\pi_{n, j} \sim \frac{c_{j, 2}\left(x_{d o m}\right)}{\Gamma(1 / 2)\left(x_{d o m}\right)^{n+1}} n^{1 / 2-1}=\frac{c_{j, 2}\left(x_{d o m}\right)}{\sqrt{\pi}} n^{-1 / 2}\left(\frac{1}{x_{d o m}}\right)^{n+1} .
$$

Case 3: According to Lemma 5.1 and Lemma $5.3, j=0, \pm 1, \pm 2, \ldots$,

$$
\varphi_{j}^{\prime}\left(\frac{x}{x_{d o m}}\right) \sim c_{j, 2}\left(x_{d o m}\right)\left(1-\frac{x}{x_{d o m}}\right)^{-1 / 2} \text { as } \frac{x}{x_{d o m}} \rightarrow 1 \text { in } \triangle \text {. }
$$

Thus, from Remark 1.3,

$$
\pi_{n, j} \sim \frac{c_{j, 3}\left(x_{d o m}\right)}{\Gamma(1 / 2)\left(x_{d o m}\right)^{n}} n^{1 / 2-2}=\frac{c_{j, 3}\left(x_{d o m}\right)}{\sqrt{\pi}} n^{-3 / 2}\left(\frac{1}{x_{d o m}}\right)^{n}
$$




\section{Chapter 6}

\section{Conclusion}

In this thesis, we considered exact tail asymptotics of stationary probabilities for a longer-queue-serve-first preemptive queue model, which is formulated as a continuous time Markov chain in the half plane. We concluded that there are three types of exact tail asymptotics along the $x$-direction: exact geometric, geometric multiplied by a prefactor of $n^{-\frac{1}{2}}$, and geometric multiplied by a prefactor of $n^{-\frac{3}{2}}$. Along the $y$-direction (either positive or negative direction), the only type is exact geometric multiplied by $|n|^{i}$.

Possible future research on this model includes:

1. Approximation of stationary probabilities of $\left\{\left(L_{1}(t), L_{2}(t)\right)\right\}$, and some queueing measures, which could be obtained based on the results in this thesis.

2. Rare event simulation of this model, which could be used to evaluate the efficiency of our results.

3. Exact tail asymptotic properties for random walks allowing bulk arrivals and bulk services, and for multi-dimensional random walks, which are generalizations of the model studied in this thesis. 


\section{Bibliography}

[1] Banderier, C., Bousquet-Mélou, M, Denise, A, Flajolet, P., Gardy, D. and Gouyou-Beauchamps, D. (2002) Generating functions of generating trees, Discrete Math., 246, 29-55.

[2] Fayolle, G., Iasnogorodski, R. and Malyshev, V. (1999) Random Walks in the Quarter-Plane, Springer, New York.

[3] Li, H. and Zhao, Y.Q. (2009) Exact tail asymptotics in a priority queuecharacterizations of the preemptive model, Queueing Systems, 63, 355-381.

[4] Li, H. and Zhao, Y.Q. (2011) Exact tail asymptotics in a priority queuecharacterizations of the non-preemptive model, Queueing Systems, 68, 165-192.

[5] Li, H. and Zhao, Y.Q. (2011) Tail asymptotics for a generalized two demand queueing model - A kernel method, Queueing Systems, 69, 77-100.

[6] Li, H. and Zhao, Y.Q. (2011) A kernel method for exact tail asymptotics random walks in the quater plane, submitted.

[7] Li, H. and Zhao, Y.Q. (2011) Analysis of exact tail asymptotics for singular random walks in the quarter plane, submitted.

[8] Lang, S. (1999) Complex analysis, Springer, New York.

[9] Miyazawa, M. (2011) Light tail asymptotics in multidimensional reflecting processes for queueing networks, Top. 19 233-299. 
[10] Bender, E. (1974) Asymptotic methods in enumeration, SIAM Review, 16, 485513.

[11] Flajolet, F. and Sedgewick, R. (2009) Analytic Combinatorics, Cambridge University Press.

[12] Flatto, L. and McKean, H.P. (1977) Two queues in parallel, Comm. Pure Appl. Math., 30, 255-263.

[13] Flatto, L. and Hahn, S. (1984) Two parallel queues created by arrivals with two demands I, SIAM J. Appl. Math., 44, 1041-1053.

[14] Flatto, L. (1985) Two parallel queues created by arrivals with two demands II, SIAM J. Appl. Math., 45, 861-878.

[15] Flatto, L. (1989) The Longer Queue Model, Probability in the Engineering and Informational Sciences, , 3, 537-559.

[16] COHEN, J.W. (1987) A two-queue, one-server model with priority for the longer queue Queueing Systems., 2, 261-283.

[17] Adan, I.J.B.F. G.J. Van. Ivo Adan. Der, Wal J. Van. (1997) The symmetric longest queue system, Stochastic Models., 13, 105-120.

[18] Zheng, Y. S. and Zipkin, P. (1990) A queuing model to analyze the value of centralized inventory information, Operations Research., 38, 296-307.

[19] Zipkin, P. (1995) Performance analysis of a multi-item production-inventory system under alternative policies, Management Science., 41, 690-703.

[20] Flajolet, P. and Odlyzko, A. (1990) Singularity analysis of generating functions, SIAM J. Disc. Math., 3, 216-240. 
[21] Kobayashi, M. and Miyazawa, M. (2011) Tail asymptotics of the stationary distribution of a two dimensional reflecting random walk with unbounded upward jumps, submitted. 